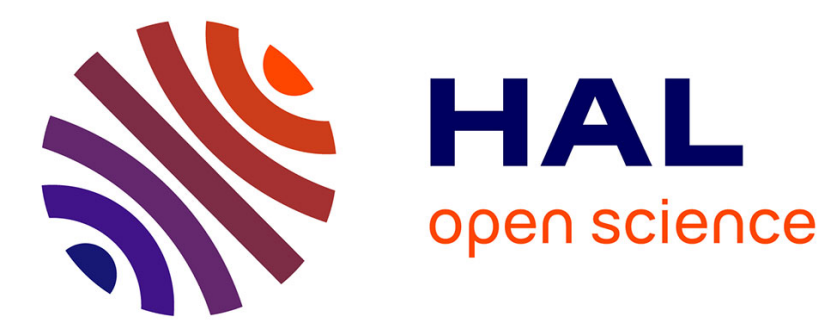

\title{
Constitutive modeling of a SEBS cast-calender: Large strain, compressibility and anisotropic damage induced by the process
}

Anne-Sophie Caro-Bretelle, Patrick Ienny, Romain Léger

\section{To cite this version:}

Anne-Sophie Caro-Bretelle, Patrick Ienny, Romain Léger. Constitutive modeling of a SEBS castcalender: Large strain, compressibility and anisotropic damage induced by the process. Polymer, 2013, 54 (17), pp.4594-4603. 10.1016/j.polymer.2013.06.006 . hal-01771587

\section{HAL Id: hal-01771587 \\ https://hal.science/hal-01771587}

Submitted on 16 May 2018

HAL is a multi-disciplinary open access archive for the deposit and dissemination of scientific research documents, whether they are published or not. The documents may come from teaching and research institutions in France or abroad, or from public or private research centers.
L'archive ouverte pluridisciplinaire HAL, est destinée au dépôt et à la diffusion de documents scientifiques de niveau recherche, publiés ou non, émanant des établissements d'enseignement et de recherche français ou étrangers, des laboratoires publics ou privés. 


\title{
Constitutive modelling of a SEBS cast-calender: large strain, compressibility and anisotropic damage induced by the process
}

\author{
A.S. Caro-Bretelle, P. Ienny, R. Leger, \\ C2MA, Ecole des Mines d'Alès, 6 Avenue de Clavières, 30319 Alès (France)
}

This article has been first published online in POLYMER in June 1, 2013.

https://doi.org/10.1016/j.polymer.2013.06.006

\begin{abstract}
The anisotropy induced through the calendering process was investigated in cyclic and monotonic uniaxial and biaxial tests monitored using an optical device. Thin sheets of Styrene-ethylene-co-butylene-styrene (SEBS) were extruded, which resulted in significant anisotropy in the polymer: the polymer chains are principally uniaxially oriented in the same direction as the calendering direction. Cyclic uniaxial tests reveal the Mullins effect and compressibility, which are higher in the calendering direction. A successful physically based hyper-elastic model is proposed, which includes anisotropic damage in the framework of continuum damage mechanics at large strains. Two sets of experimental data (samples cut in the calendering direction and in the transverse direction) are used to validate the constitutive model. Model parameters are estimated via scripts written in Matlab®. Computational results agree rather well with experimental data, and highlight the influence of process on material properties. Model capabilities for predicting various test results (samples cut in the diagonal direction and tensile tests on a two-hole specimen) are critically discussed.
\end{abstract}

Keywords: damage, digital image correlation, hyperelasticity 


\section{Introduction}

In this paper the effect of planar anisotropy induced by the forming process is modelled. The two most important manufacturing methods with thermoplastic elastomers are extrusion and injection molding. Fabrication via injection molding is extremely rapid and highly economical. Both equipments and methods usually used for extrusion or injection molding of a conventional thermoplastic are generally suitable for TPEs. TPEs can also be processed by blow molding, thermoforming, and heat welding. In this paper, extrusion followed by injection molding or dip coating processes are used to produce thin films with a controlled thickness. Once the material is compounded, it is either shaped into sheets by flat path calender or by dip coating. In the first process polymers chains are uniaxially oriented, yielding pronounced structural anisotropy; on the contrary dip coating produces isotropic films.

The present study focuses on the mechanical behavior of a Styrene-Ethylene-coButylene-Styrene for which high deformability, compressibility and strain softening are relevant assumptions. This kind of polymer is widely used in industry because of its low cost, optical transparency, biocompatibility and flexibility. Its complex behavior is known and usually studied through filled materials [1-3]. Nevertheless some authors modeled unfilled silicone rubber [4] but all of these studies assume that this material is isotropic.

A phenomenological model developed in the framework of continuum damage mechanics is proposed. Among numerous modeling $[5,6]$, high deformability and compressibility are modeled via a Rivlin phenomenological approach [7]. Strain softening is assumed to be anisotropic and derives from Miehe's theory [8, 9].

In order to identify parameters of the model, an experimental work including cyclic uniaxial tensile tests, biaxial and compression tests is performed.

The outline of this article is as follows. After the experimental section (2) mechanical tests results are discussed (3) to propose a phenomenological model suitable to reproduce experimental results (4). Throughout the article, various samples, cut in different directions in the plane of the sheet, are tested in order to study the influence of process on mechanical properties. It will be shown that the calender process induces an increase of both stress and damage in the calender direction in comparison with the transverse one. Parameter's optimization is presented in section (5) for samples cut in the calender and transverse directions and from uniaxial, biaxial and Oedometric tests. Finally, the model has been 
validated for a sample cut in the diagonal direction. Lastly, in order to test the ability of the model to describe strain and stress of complex structure, a sample sheet containing two holes is uniaxially loaded. Finite elements simulation of this test is then performed and compared to experimental results.

\section{Experimental}

\subsection{Materials and preparation}

The SEBS triblock copolymer used in this study has been purchased at Plastic Technology Service LTD (reference SEBS Thermoflex 10H730). This system is characterized by an 11 shore A hardness, a low amount of polystyrene (PS) 5\% by weight in its end block and by a ratio of butylene to ethylene less than one (30\%wt butylene). This composition allows a very low crystallinity degree (less than 1\%). Structures of these kinds of block polymers (or similar) has been widely studied especially when structure-property correlations are expected [10-12].. PS domains act as glassy phase with physical crosslinks while PEB are elastomeric blocks which give the rubbery behavior [13]. Depending on both the amount of PS and the ratio of butylene to ethylene the PS domains coalesce into cylindrical or lamellar rods packed in a two-dimensional hexagonal lattice [10], spherical domains embedded within the PEB matrix [11].

SEBS has been casted as films with a cast-extruder Polylab OS / Rheomex 19/25 of Thermofisher Company. This device is equipped with a $30 \mathrm{~cm}$ wide flat-path to control the thickness from $0.2 \mathrm{~mm}$ to $1.2 \mathrm{~mm}$ and a cast-conveyor that helps cooling the film to control its crystallinity. The film is then rolled. Such process creates films with anisotropic properties which are mostly caused by preferred orientations of polymer chains developed during the severe plastic deformation such as cold rolling [11, 12].

Other films have been prepared by solubilisation process. SEBS was first dissolved in xylene to prepare a $0.3 \mathrm{wt} \%$ solution. This solution was then deposited onto a flat support and let few hours under a hood at ambient temperature to evaporate the solvent. This sequence is repeated until obtaining the desired thickness of deposit. The film was annealed for $2 \mathrm{~h}$ in a vacuum oven at $140{ }^{\circ} \mathrm{C}$.

Properties of casted films have been evaluated in the longitudinal direction (Calender Direction: $\mathrm{CD}$ ), in the transverse direction (TD) or in the diagonal direction $\left(45^{\circ}\right)$ of the roll. The longitudinal direction corresponds to the extrusion direction; the transverse direction 
corresponds to the direction parallel to the width of the extruded film, at right angles to the length of the roll and the diagonal direction is at $45^{\circ}$ to the length of the roll (Fig. 1).
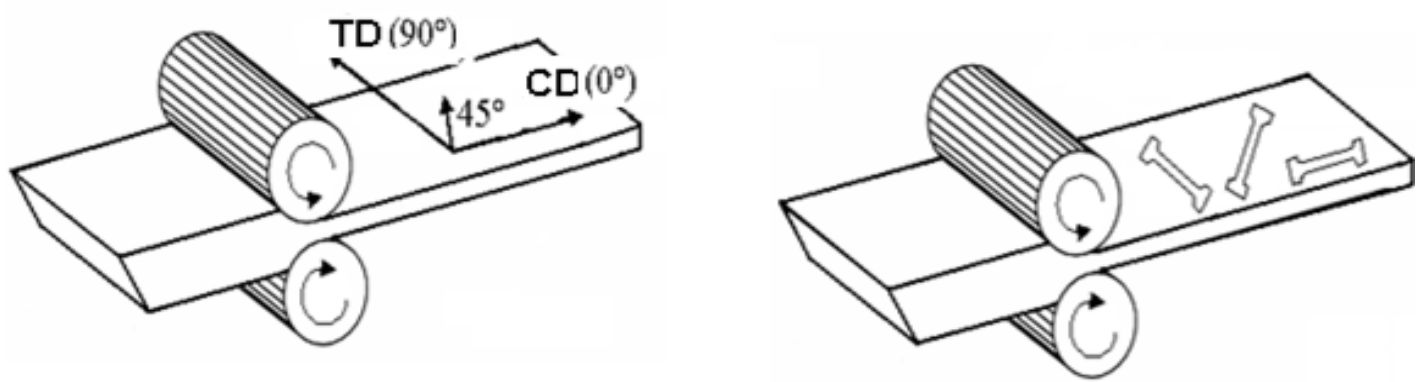

Fig. 1: Anisotropy induced by the process

Rectangular and discs shape specimens of a $0.6 \mathrm{~mm}$ thickness were cut from films (see Fig. 2):

- Rectangular strips (height $L=60 \mathrm{~mm}$, width $l=30 \mathrm{~mm}$ corresponding to the useful dimensions, see (a) in Fig. 2) were cut along the longitudinal, transverse and diagonal directions of the calender direction. Samples have been produced for both manufacturing process. An uniaxial load is applied to these samples.

- Rectangular strips (height $L=10 \mathrm{~mm}$, width $l=56 \mathrm{~mm}$ corresponding to the useful dimensions, see (b) in Fig. 2); a uniaxial loading on these samples enables a biaxial solicitation in which transverse strain is prevented.

- Disc shape specimens (diameter $d=20 \mathrm{~mm}$ and height $h=10 \mathrm{~mm}$, see (c) in Fig. 2): thin disks were stamped and stacked together to achieve a compression cylinder of total height $h$; oedometric compression tests were performed on these specimens to enhance the material compressibility.

- A rectangular shape specimen with two holes (see (d) in Fig. 2) was cut and loaded uniaxially to produce heterogeneous strains; a prior finite element simulation enabled the definition of this geometry to maximize shearing strain between the two holes of the specimen. 

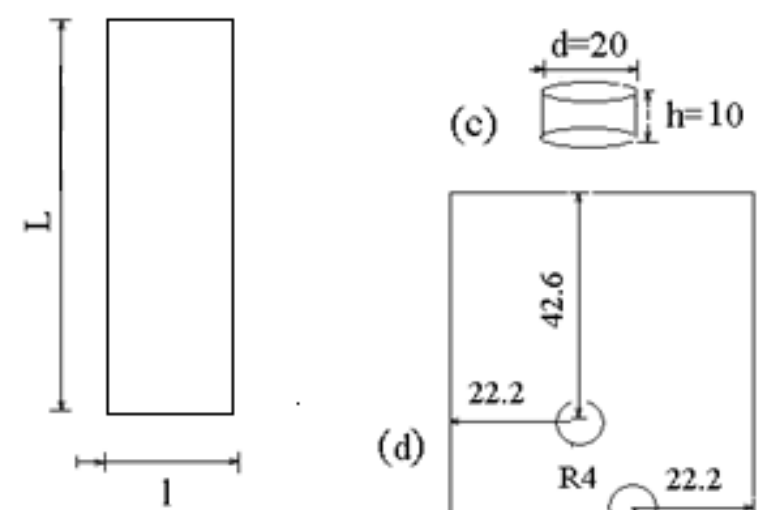

(d)

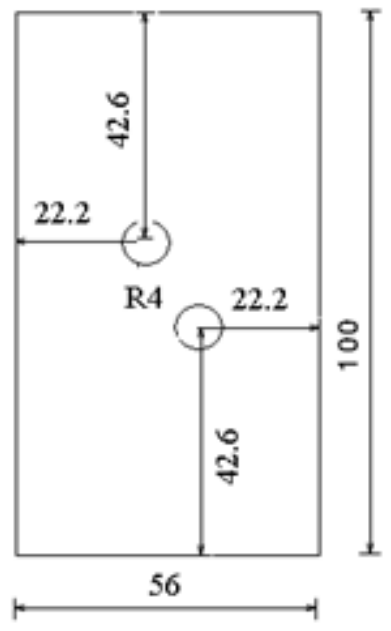

(b) $L=10 \mathrm{~mm}, \mathrm{l}=56 \mathrm{~mm}$

Fig. 2: Useful dimensions of specimens: for uniaxial (a), biaxial (b) compression loadings (c) and heterogeneous test (d) (quotations in mm, thickness $0.6 \mathrm{~mm}$ )

\subsection{Quasi-static tests}

Let $(\boldsymbol{x}, \boldsymbol{y}, \boldsymbol{z})$ and $(\boldsymbol{C D}, \boldsymbol{T D}, \boldsymbol{z})$ be two cartesian bases, attached to the device and to the material respectively (Fig. 3), $\boldsymbol{x}$ is the tensile direction, $\boldsymbol{y}$ is perpendicular to $\boldsymbol{x}$ and in the planar surface and $z$ the out-of-plane direction. The $\theta$-angle qualifies the material direction: $\theta=(\boldsymbol{x}, \boldsymbol{C D})\left(\theta=0^{\circ}\right.$ corresponds to the $\mathrm{CD}$ direction, $\theta=90^{\circ}$ to the TD direction and $\theta=45^{\circ}$ to the diagonal direction). As usual, both basis are related by the relation: $(x, y, z)^{t}=$ $\boldsymbol{P}(\boldsymbol{C D}, \boldsymbol{T D}, z)^{\mathrm{t}}$ where $\boldsymbol{P}=\cos (\theta)(\boldsymbol{C D} \otimes \boldsymbol{C D}+\boldsymbol{T D} \otimes \boldsymbol{T D})+\sin (\theta)(\boldsymbol{C D} \otimes \boldsymbol{T D}-\boldsymbol{T D} \otimes \boldsymbol{C D})+\boldsymbol{z} \otimes \boldsymbol{z}$ and the superscript ${ }^{\mathrm{t}}$ denoted the transpose operator. 


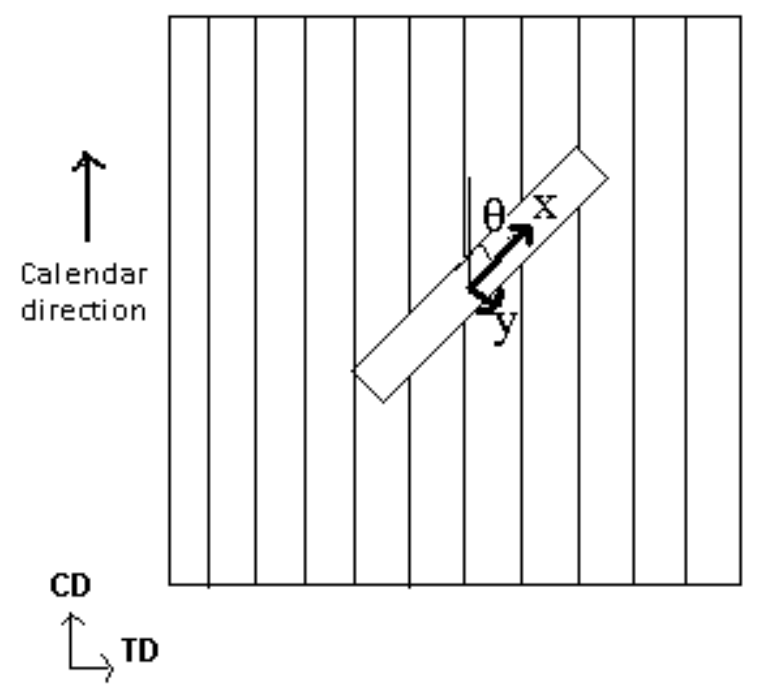

Fig. 3: Material and device frames

\subsubsection{Mechanical loadings}

Uniaxial tensile tests were conducted on a Zwick TH010 universal testing machine. The crosshead speed was adapted to have the same longitudinal strain rate for the tensile and biaxial tests. The tensile test is conducted up to a defined nominal strain. The software used is TestXpert II ${ }^{\circledR}$ and allows the recording of time, load $(F)$ and stretch. In order to analyse the mechanical behavior, samples were subjected to several homogeneous loadings and one heterogeneous.

- Uniaxial tensile tests were carried out on rectangular strip specimens at room temperature (see Fig. 2). These tests were performed with crosshead speeds of $10 \mathrm{~mm} / \mathrm{min}$ and $100 \mathrm{~mm} / \mathrm{min}$. During the test, nominal stress (first Piola-Kirchhoff) $\boldsymbol{\sigma}=\sigma_{x x} \boldsymbol{x} \otimes \boldsymbol{x}$ and deformation gradient tensor $\boldsymbol{F}=\lambda_{x} \boldsymbol{x} \otimes \boldsymbol{x}+\lambda_{y} \boldsymbol{y} \otimes \boldsymbol{y}+\lambda_{z} z \otimes z+\lambda_{x y} \boldsymbol{x} \otimes \boldsymbol{y}$ are assumed both to be homogeneous and to retain this form during the test even at high strain level. The axial stress $\sigma_{x x}=F / L l$ was obtained by the load cell and the in-plane stretch ratios $\lambda_{x}=L^{\prime} / L, \quad \lambda_{y}=l^{\prime} / l, \quad \lambda_{x y}$ were deduced from DIC. Measurements from a digital calliper enables also the values of $\lambda_{z}=h^{\prime} / h$ (inaccessible out-of-plane measurement) provided stopping temporarily the test; the subscript' is relative to the deformed state. Reproducibility tests on a CD sample are shown in Fig. 4 where nominal stress is plotted versus stretch ratios. DIC was performed on tests 
with or without stopping (DIC1 and DIC2 respectively); associated results confirm the reproducibility (see Fig. 4 in which $\lambda_{x \mathrm{DICl} 1}, \lambda_{x \mathrm{DIC} 2}$ and $\lambda_{y \mathrm{DICl} 1}, \lambda_{y \mathrm{DIC} 2}$ (values of $\lambda_{x}$ issued from DIC) correspond). A comparison between $\lambda_{z}$ measured with the calliper (denoted $\lambda_{z \text { Calliper }}$ ) and the transverse stretch ratio $\lambda_{y}$ obtained by DIC ( $\lambda_{y \mathrm{DIC} 1}$ or $\left.\lambda_{y \mathrm{DIC} 2}\right)$ is drawn. These results validate DIC (stretches ratios deduced from DIC and measured by digital calliper correspond) and demonstrate transverse isotropy for the CD sample $\left(\lambda_{y}=\lambda_{z}\right)$. This transverse isotropy assumption was validated in a previous article on polycarbonate nanocomposite specimen submitted to uniaxial tensile test

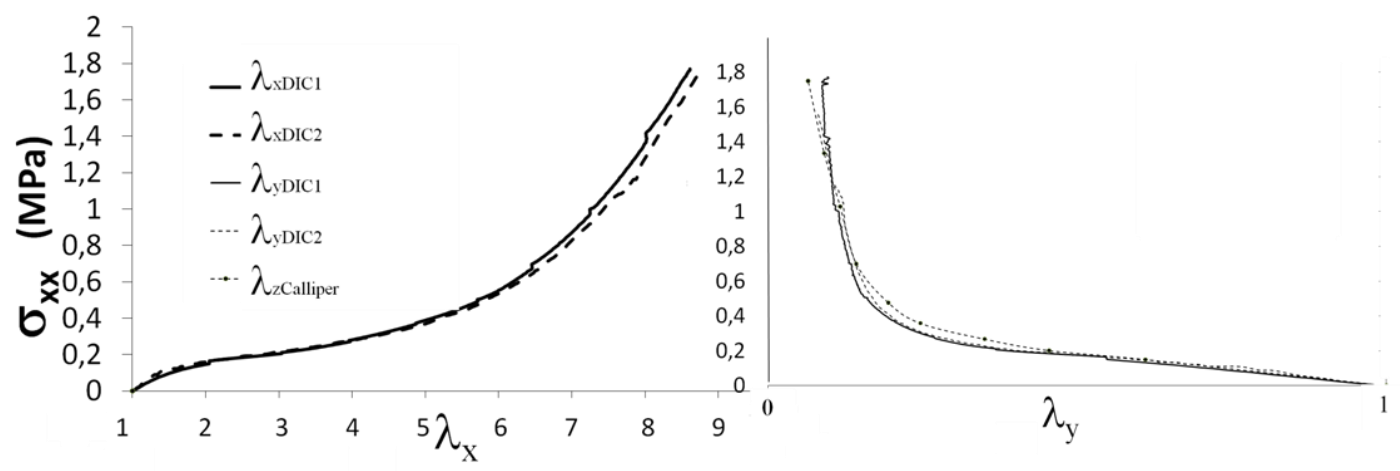

Fig. 4: Uniaxial stress as function of measured stretch ratios (measured by DIC and calliper device)

In Fig. 5 uniaxial stress $\sigma_{x x}$ versus stretches ratios $\lambda_{x}, \lambda_{y}$ are reported for calender and dip coated samples tested for both displacement speeds $(10 \mathrm{~mm} / \mathrm{min}$ and 100 $\mathrm{mm} / \mathrm{min}$ ). First of all the anisotropy induced by the process is clearly demonstrated: the response is stiffer in the direction of calendering. The comparison between the two processes shows a significant reduction of stiffness beyond $500 \%$ strain for the material processed by solubilisation. Otherwise and unexpectedly, the level of stress slightly increases with a decreasing strain rate: increasing strain rate should therefore induce a greater damage excepted for the dip coated samples for which the curves are superimposed. Nevertheless, the small effect of the time dependence behavior has led us to neglect this phenomenon for this study. 


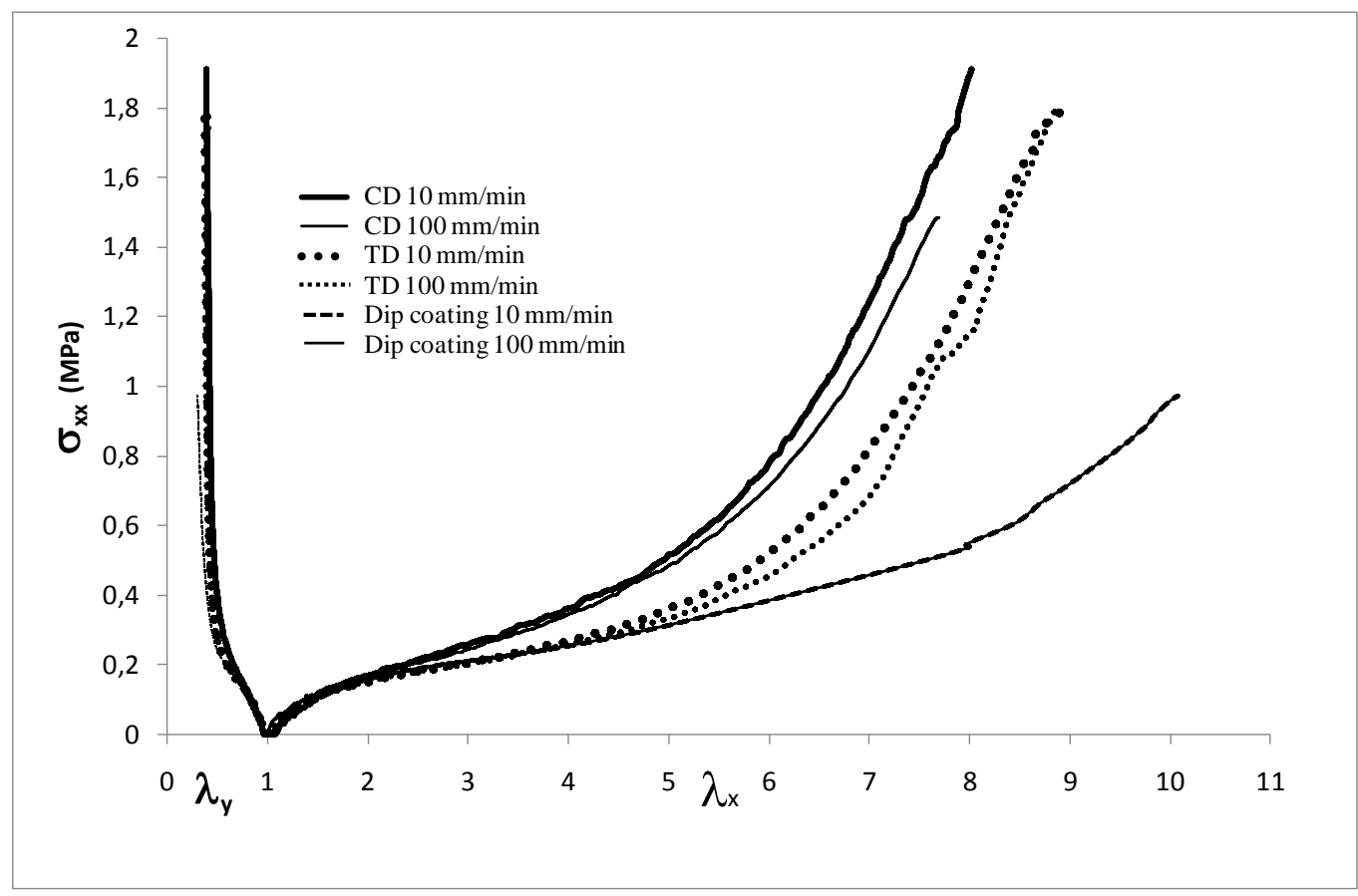

Fig. 5: Uniaxial stress versus stretches ratios for SEBS manufactured by a calender process (CD, TD) and by dip coating process

Finally uniaxial cyclic tensile tests are performed on CD and TD specimens. They allow a relevant material identification of damage parameters.

- Biaxial tensile tests were conducted on rectangular strip specimen at crosshead speeds of $1.66 \mathrm{~mm} / \mathrm{min}$. The same equipment as for the tensile test was used. The load cell gives access to the axial force $F$ which leads to the estimation of the component $\quad \sigma_{x x}=F / L l \quad$ of the stress tensor: $\boldsymbol{\sigma}=\sigma_{x x} \boldsymbol{x} \otimes \boldsymbol{x}+\sigma_{y y} \boldsymbol{y} \otimes \boldsymbol{y}$. The deformation gradient tensor $\boldsymbol{F}=\lambda_{x} \boldsymbol{x} \otimes \boldsymbol{x}+\lambda_{y} \boldsymbol{y} \otimes \boldsymbol{y}+\lambda_{z} z \otimes z+\lambda_{x y} \boldsymbol{x} \otimes \boldsymbol{y} \quad$ is homogeneous and can be simplified using the assumption $\lambda_{y}=1 . \lambda_{x}, \lambda_{y}, \lambda_{x y}$ are obtained from DIC while $\lambda_{z}$ and $\sigma_{y y}$ are unknown but can be deduced from theory and experiments (see section 3).

- A tensile test was also performed on a rectangular planar specimen containing 2 holes as shown in Fig. 2. The test was conducted with the same equipment: the load cell measure the axial force $F$ and DIC gave the local in-plane stretches which is, in this case, heterogeneous.

- An oedometric device was also developed to evaluate the bulk modulus, necessary parameter to describe material compressibility. Samples (see Fig. 2) are subjected 
to compressive load in a cylinder expected to be undeformable. Load and displacement cells enable the evaluation of bulk modulus.

\subsubsection{Strain field measurements}

During the mechanical tests, the deformations were measured by an optical extensometer including a high resolution Charge camera (Redlake Megaplus 2, 1920 by 1080 pixels, coded in 256 grey levels). A vertical translation table unit was used to follow the region of interest (ROI) of the tested specimen. The image acquisition is controlled by a LabVIEW $^{\circledR}$ software which allows the simultaneous record of images and data from the testing machine (such as load and crosshead displacement). The scale factor ( $\mathrm{k}=78 \mu \mathrm{m} / \mathrm{pixel}$ for homogeneous tests and $\mathrm{k}=37 \mu \mathrm{m} / \mathrm{pixel}$ for the heterogeneous test) is fixed to constitute a compromise between a necessary spatial resolution and the limitation of the perspective errors associated to the out of plane displacement. Different scale factors were used for both types of test on homogeneous and heterogeneous specimens $[14,15]$. To obtain a signature for the correlation treatment, some carbon powder is deposited on the observed surface of the specimen. Digital Image Correlation software CinEMA (produced by the Alès School of Mines) allows the determination of the two-dimensional displacement components with a subpixel accuracy: from two treated images, a correlation calculation is made at every meshing point (Fig. 6). Each point of the virtual mesh corresponds to the centre of a pattern. This pattern, defined by its size denoted CS (for correlation size), is the representative area of the material point which is tracked. Distance between mesh points is given by the Gs (for Grid step) parameter. Deformation calculation is obtained by the first order derivation of the displacement field around the considered point. The dimension of the area where is evaluated the in-plane gradient tensor is fixed by the Ns (for Number of Steps) parameter which qualify the number of points to take into account in the vertical and horizontal directions, both directions being equivalent from this point of view. Of course, associated with the magnification, these variables characterize the bidimensional Gage Lenth (GL) expressed in pixel or mm: $\left(\mathrm{GL}=2 \cdot \mathrm{N}_{\mathrm{s}} \cdot \mathrm{G}_{\mathrm{s}}+\mathrm{C}_{\mathrm{s}}\right)$. The choice of these parameters should guarantee a good accuracy without losing local information and should reduce errors and the computation time (Table 1).

Table 1: Data parameter used by the DIC Process 


\begin{tabular}{cccccc}
\hline Specimen & $\begin{array}{c}\text { Acquisition } \\
\text { speed }\end{array}$ & $\begin{array}{c}\text { Cs } \\
\text { (pix/mm) }\end{array}$ & Ns & $\begin{array}{c}\text { Gs } \\
\text { (pix/mm) }\end{array}$ & $\begin{array}{c}\text { GL } \\
\text { (pix/mm) }\end{array}$ \\
\hline unnotched specimen & $1 \mathrm{im} / \mathrm{s}$ & $30 / 2.34$ & 5 & $30 / 2.34$ & $330 / 25.2$ \\
\hline $\begin{array}{c}\text { Heterogeneous } \\
\text { specimen }\end{array}$ & $1 \mathrm{im} / \mathrm{s}$ & $30 / 1.11$ & 1 & $20 / 0.74$ & $70 / 2.59$ \\
\hline
\end{tabular}

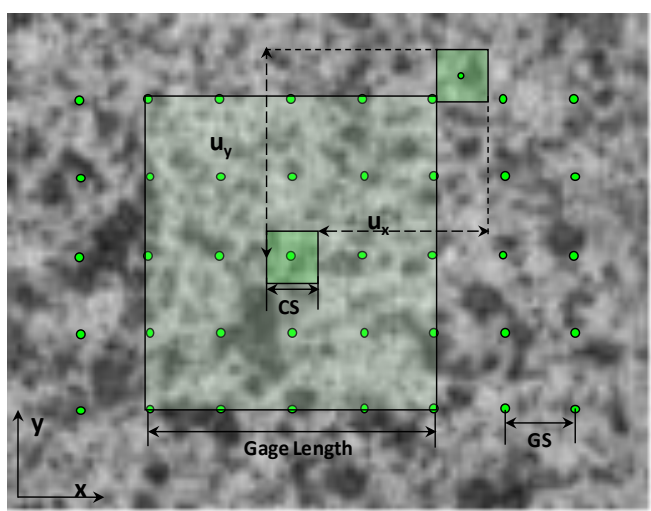

Fig. 6: Definition of the two-dimensional digital extensometer (CS: correlation size; GS: grid step

The number of steps (or images) required to reach a given strain state calculation induces a greater or lesser cumulative errors on strain. For the processing mode used in the present study and considering both the resolution on displacements (Standard Deviation $=10^{-2}$ pixels) and the virtual mesh used for the homogeneous and heterogeneous tests, the cumulative error does not exceed $10^{-4}$ and $10^{-3}$ respectively to the level of strain considered $[14,15]$ After evaluating of the two-dimensional fields of in-plane displacement a linear estimation of the displacement gradient realized on several points chosen around the studied point allows the calculation of the deformation gradient tensor.

\section{Homogeneous tests}

\subsection{Cyclic behavior, material anisotropy}

Uniaxial cyclic tensile tests are then performed on CD and TD specimens: they consist in achieving first three loading-unloading up to the axial stretch level of about $\lambda_{x l}=3.7$ and then successively up to about $\lambda_{x 2}=5.3$ and about $\lambda_{x 3}=6.6$ of the axial stretch. Nominal stress- 
strain curves are shown in Fig. 7 for both samples. They behave as classical elastomer presenting a stress softening depending on the material direction. We observe a significant reduction on the stress at a given level of strain for loading sequences which follows the first loading, phenomenon known as the Mullins effect [16]. Moreover both specimens show a relatively low level of hysteresis, which is already stabilized after the second cycle. These observations are consistent with a low level of crystallinity supposed previously: this type of elastomer does not crystallize under loading [10]. Regarding to the low level of PS incorporation, the permanent set never exceeds $\lambda_{x}=1.4$ (40\% of strain) and does not change beyond the first cycling. For the modeling, we can consider this value to be negligible with respect to the maximum elongation undergone by the material during the test $\left(\lambda_{x}>10\right)$. This result was also expected since this permanent set seems to be linked with the level of PS content [10].

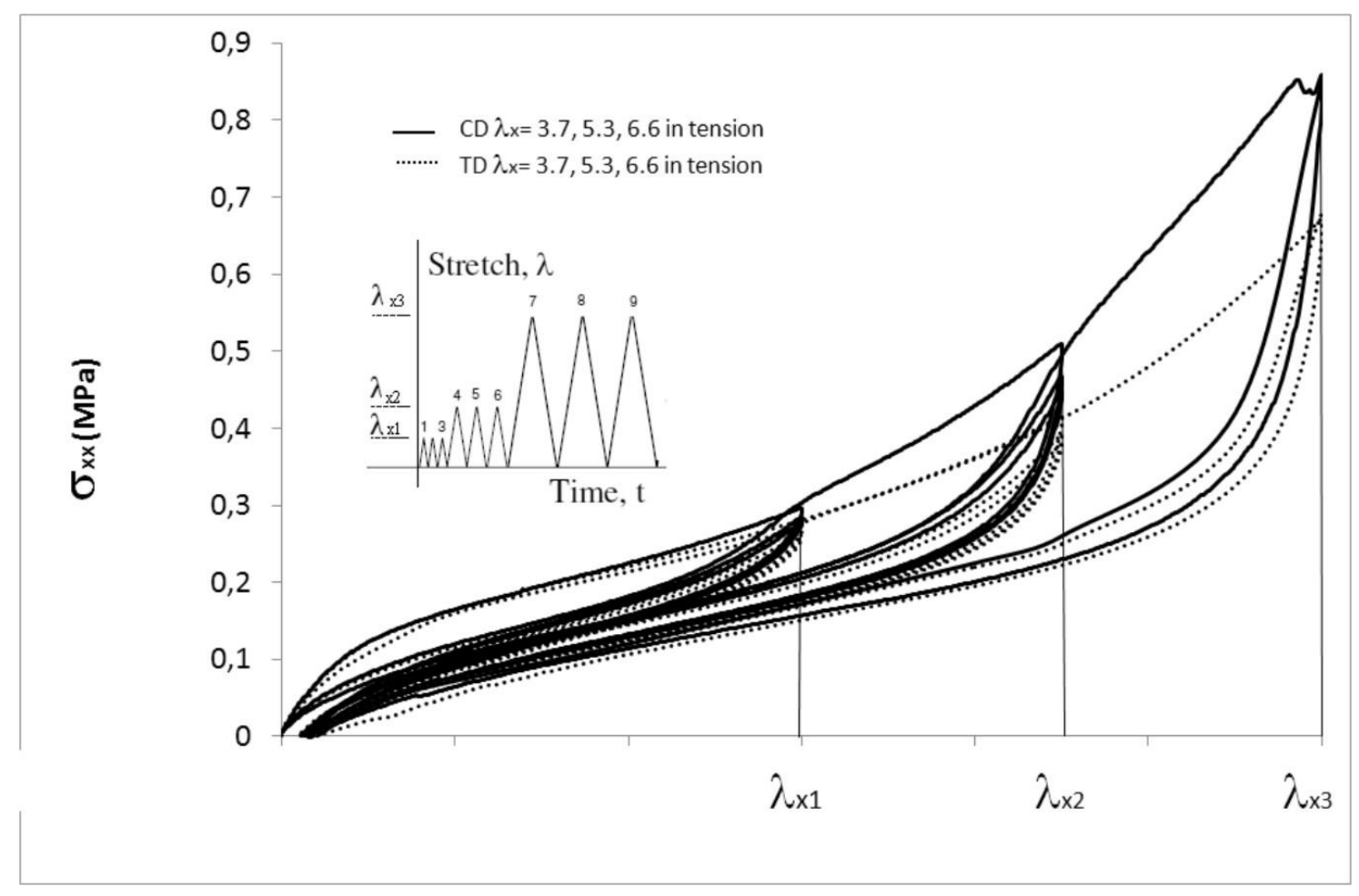

Fig. 7: Stress-Stretch response during three cycles of loading-unloading at 3.7, 5.3 and 6.6 of stretch (for CD and TD samples)

It is possible to record volume strain $\mathrm{E}_{V}$ up to large deformations, where $\mathrm{E}_{V}$ is defined as:

$\mathrm{E}_{V}=\lambda_{x} \lambda_{y} \lambda_{z}-1$. 
In the case of uniaxial tensile test performed in calender direction and under transverse isotropy assumption (see section 2.2.2), this volume strain can be deduced from surface measurements: $\mathrm{E}_{V}=\lambda_{x} \lambda_{y}^{2}-1$. This volume variation increases first according to the growth of damage and decreases after 5 of stretch level (see "primary loading" in Fig. 8). This phenomenon has been already observed previously and can be attributed to a decrease of strain speed while the macro-molecular chains are aligned in the loading direction or the beginning of crystallization [17]. As commonly observed in the literature [18], this volume strain trend depends on mechanical cycling (see Fig. 8). While successive loadings lead to the same volume fraction evolution (as a function of longitudinal strain), the curves are gradually shifted to lower volume strains. It is important to note that, following an initial loading, the material is in compression state for a zero load level. This is probably due to a reorganization of the material microstructure under loading.

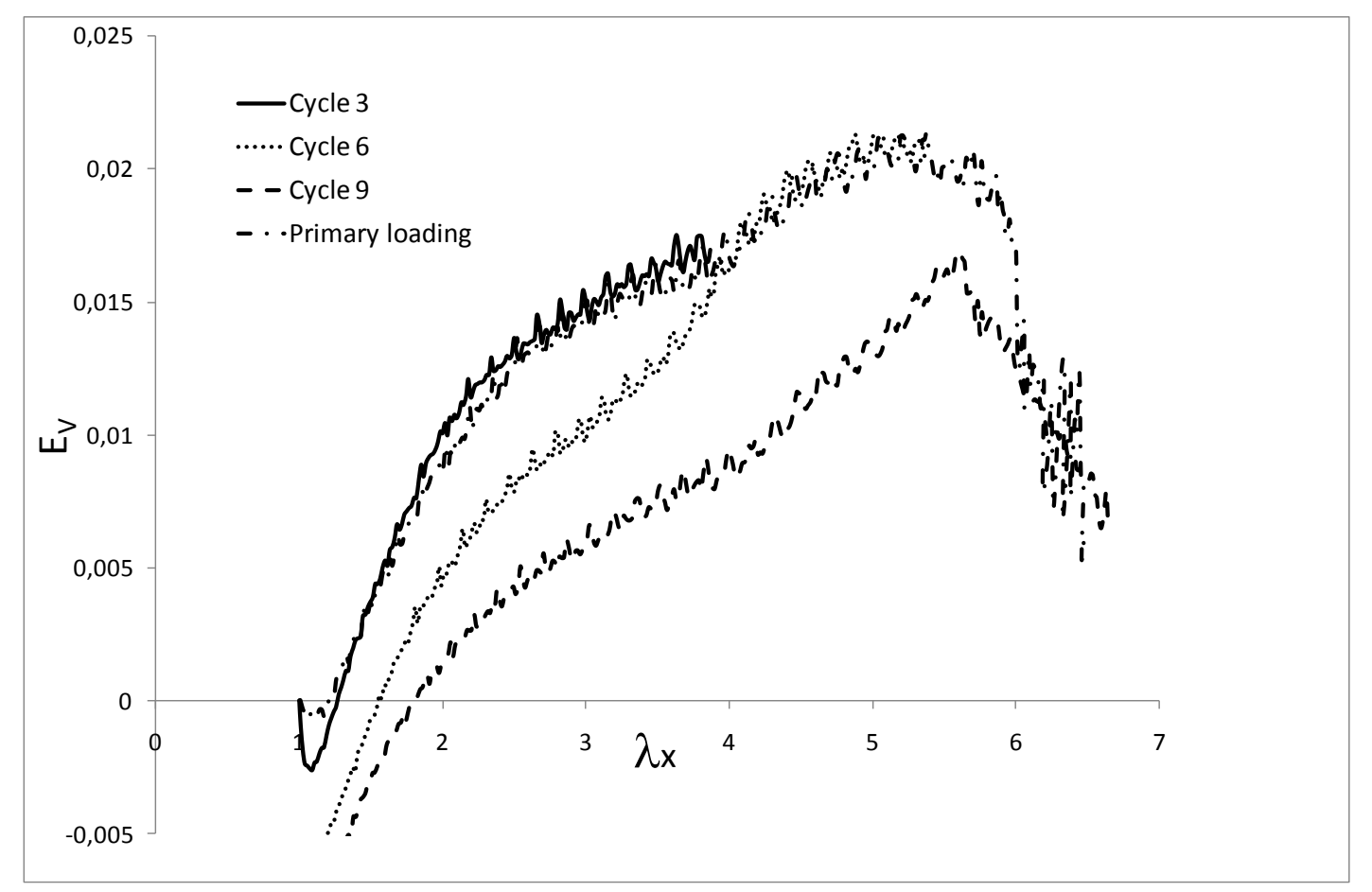

Fig. 8: Volumetric variation as a function of mechanical cycling for a CD sample

\subsection{Biaxial test, material anisotropy}

In the graph of Fig. 9, the evolution of axial stress is reported as function of the stretch ratios. It is observed the same behavior as for the uniaxial tensile test in the same proportion. As expected, longitudinal stress for the biaxial test is greater than for uniaxial one at the same strain level. In addition to the already noted difference between the transverse and the 
calendering directions, these results first highlight the strong influence of the type of mechanical loading on stress-stretch curves and the assumption of quasi-restrained test $\left(\lambda_{y}=1\right)$ which is thus validated.

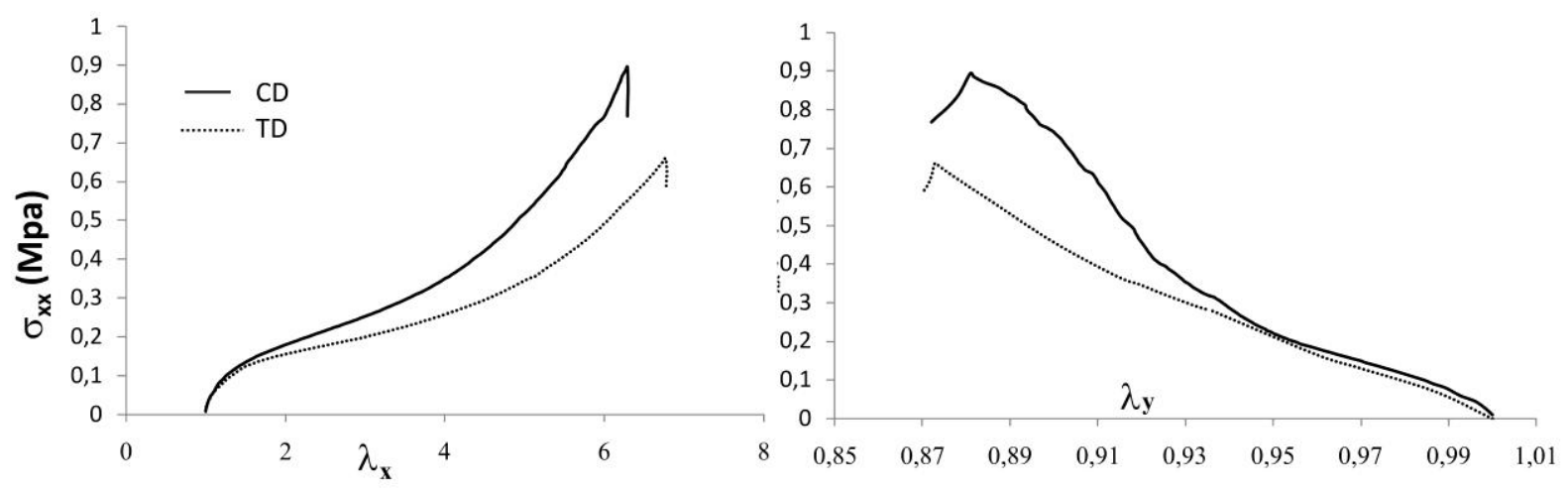

Fig. 9: Axial stress versus stretches for CD and TD specimens for a biaxial loading

\subsection{Oedometric test}

To ensure reproducibility, the tests were performed three times. The samples (Fig. 2c) are stabilized under three loading-unloading cycles successively up to $-4 \mathrm{kN},-3 \mathrm{kN},-2 \mathrm{kN}$, $1 \mathrm{kN}$ of the load. The height variation of the sample is deduced from the crosshead displacement reduced by the displacement corresponding to the machine and device stiffness. The load - displacement curve (see Fig. 10) is fitted on the stabilized loading with a linear function ( $a$ is the director coefficient) between $-3.5 \mathrm{kN}$ and $-2 \mathrm{kN},-2.5 \mathrm{kN}$ and $-1 \mathrm{kN},-2 \mathrm{kN}$ and $-0.5 \mathrm{kN},-1 \mathrm{kN}$ and $-0.5 \mathrm{kN}$. The bulk modulus is therefore deduced at each loading cycle and estimated by the expression $K=4 a h / \pi d^{2}$. The values of $K$, which increases with the hydrostatic pressure (defined by $p=\operatorname{tr}(\boldsymbol{\sigma})$ ), can be found in Table 2. In this work, assuming that the same behavior is obtained in hydrostatic tension and compression, we have to interpolate the bulk modulus for a positive hydrostatic state associated to the maximum stress met during the tests. For both uniaxial and biaxial tests, hydrostatic stress is of the order of 1 $\mathrm{MPa}$ (corresponding to $\sigma_{x x} / 3$ for uniaxial stress) and leads by interpolation to a bulk modulus value of $481 \mathrm{MPa}$. 


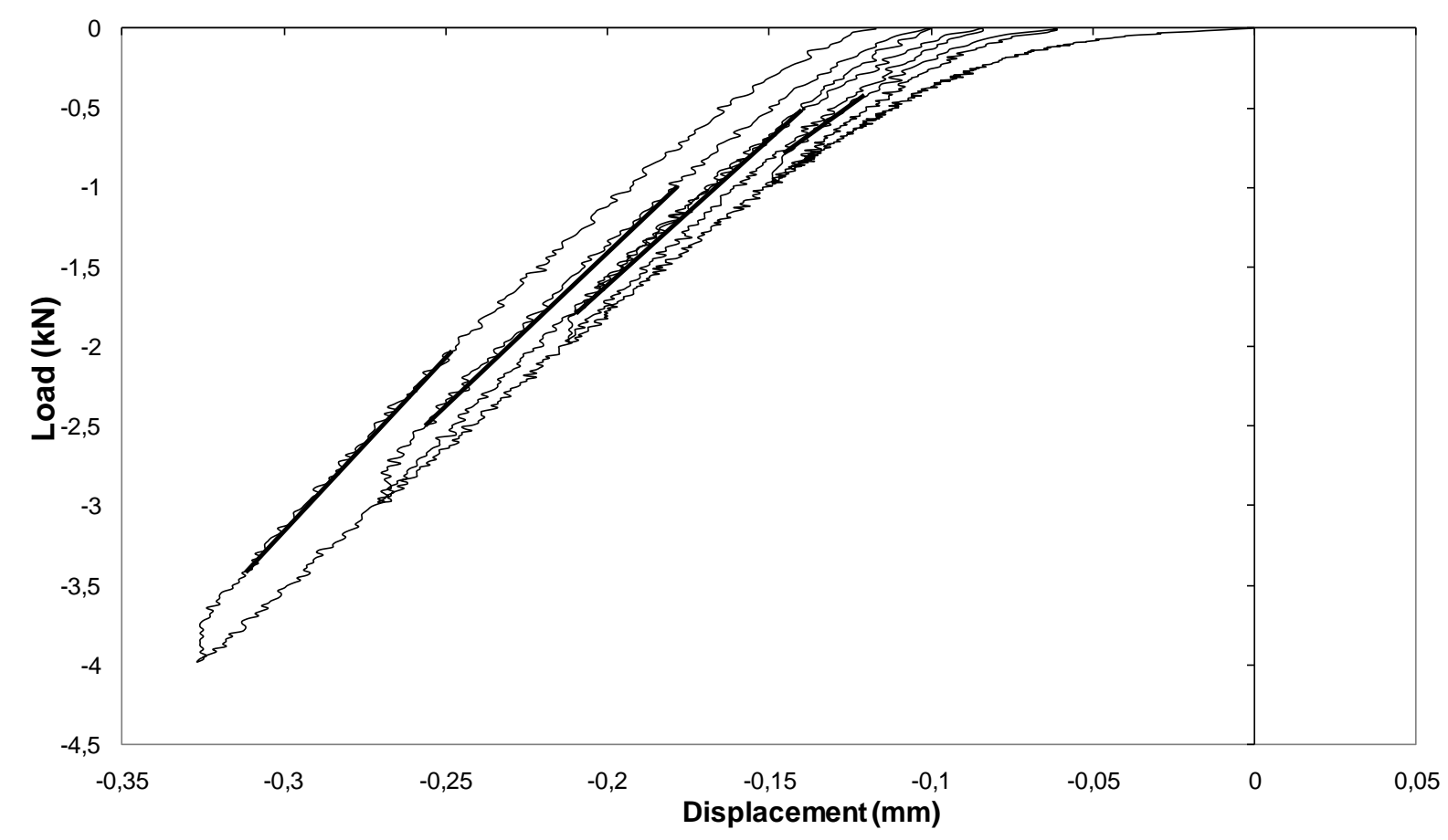

Fig. 10: Load-displacement curve in oedometric compression

Table 2: Variation of the bulk modulus with the hydrostatic pressure

\begin{tabular}{|l|l|l|l|l|}
\hline $\boldsymbol{K}$ (MPa) & 529 & 596 & 644 & 694 \\
\hline $\boldsymbol{p}$ (MPa) & 3.2 & 6.4 & 9.5 & 12.7 \\
\hline
\end{tabular}

\section{Phenomenological modeling of the mechanical behavior}

\subsection{Theoretical backgroung}

As far as our analysis is phenomenological, the type of stress-strain curves is closed to the one of crosslinked rubber. A review of molecularly based model can be found in [19]. The studied SEBS exhibits a hyperelastic behavior: elastic (recoverable) but highly nonlinear. In particular, the constitutive behavior of such materials is usually derived from the strain energy potentials with the assumption that the material is isotropic. The Cauchy stress component can be expressed in the device frame as:

$$
\boldsymbol{\sigma}=\frac{1}{J} \frac{\partial W}{\partial \boldsymbol{F}} \boldsymbol{F}^{T} \quad \text { where } J=\operatorname{det} \boldsymbol{F} .
$$

In the phenomenological approach, this energy is defined through either strain invariants $[5,7,20-22]$ or stretch ratios [23] via a polynomial development. In this study, the 
model of Rivlin [7] has been selected to describe this hyperelastic contribution. This energy enables a good approximation of the non linearity response at low and large strain because of the polynomial degree. The deviatoric part of the density energy is given by:

$W_{\text {iso }}(\boldsymbol{F})=c_{10}\left(I_{1}-3\right)+c_{01}\left(I_{2}-3\right)+c_{11}\left(I_{1}-3\right)\left(I_{2}-3\right)$

where $c_{10}, c_{01}, c_{11}$ are the material parameters and $I_{1}=\operatorname{tr}\left(\boldsymbol{F}^{t} \boldsymbol{F}\right), I_{2}=\operatorname{tr}\left(\boldsymbol{c o f}\left(\boldsymbol{F}^{t} \boldsymbol{F}\right)\right)$.

This model has been extended for compressible materials where an additive decomposition of hyperelastic potential in deviatoric and volumetric part is used:

$W(\boldsymbol{F})=W_{\text {iso }}\left(I_{1}, I_{2}\right)+W_{\text {vol }}(J)$

The compressibility component is associated with volumetric expansion while the incompressibility component is associated with volume constant distorsion. Among the various forms present in the literature (see [24] for example) we choose:

$W_{\text {vol }}(J)=\frac{K}{2}(J-1)^{2}$

where $K$ is the bulk modulus (see section 3 ).

From equation 3 to 5 the strain energy is therefore defined by:

$W(\boldsymbol{F})=c_{10}\left(I_{1}-3\right)+c_{01}\left(I_{2}-3\right)+c_{11}\left(I_{1}-3\right)\left(I_{2}-3\right)+\frac{K}{2}\left(I_{3}-1\right)^{2}$,

with $I_{3}=\operatorname{det}\left(\boldsymbol{F}^{t} \boldsymbol{F}\right)$.

From equations 2 and 6 the nominal stress can be computed as follow:

$$
\boldsymbol{\sigma}=\frac{1}{J} M_{1} B+\frac{1}{J} M_{2} B^{-1}+\frac{1}{J} M_{3} \mathbf{1}
$$

where

$M_{1}=2 \frac{\partial W}{\partial I_{1}}, M_{2}=-2 I_{3} \frac{\partial W}{\partial I_{2}}, M_{3}=2 I_{3} \frac{\partial W}{\partial I_{g}}+2 I_{2} \frac{\partial W}{\partial I_{2}}, \boldsymbol{B}=\boldsymbol{F} \boldsymbol{F}^{t}$ and $\mathbf{1}$ is the identity tensor.

Therefore, we assume that the hyperelastic part of the material behavior is isotropic unless global anisotropy is related to damage. Some authors have modeled anisotropic hyperelastic behavior using material directions decomposition [25] or additive principal invariants [26].

On the other end, sequenced tensile tests (Fig. 7) highlight a damageable behavior sensitive to the direction of calender associated to measured volume variation (Fig. 8). This softening behavior has been the subject of considerable study [27, 28]. In the literature some authors related this phenomenon to failure/slip between matrix and inclusion, inclusions failure or disentanglement of matrix macromolecules [29]. Many phenomenological theories have been proposed in the literature. Some of them are based on the two phase microstructural models introduced by Mullins and Tobin [30] and developed by Johnson and Beatty [31]. Another class of models is developed within the framework of damage mechanics and 
introduces a parameter of damage $d$ which affected the strain energy density [8, 9, 32]. For example Miehe [8] proposed an isotropic modeling via two parameters which are related to the damage velocity $(\eta)$ and to the level of damage $\left(d_{\infty}\right)$. It is common to define an effective stress from this damage parameter [33]. The stress softening is generally attributed to both breakage and shape of the PS domain [12]. Otherwise, the microstructural state is clearly not described with Miehe approach. Nevertheless this theory, which introduces a global damage parameter is simple and in accordance with our phenomenological observation. However, in order to consider micromechanical difference of conformation between CD and TD, we propose to introduce an anisotropic damage of SEBS, described through a tensorial expression $\boldsymbol{D}$ in place of the single scalar parameter commonly used :

$\sigma_{\text {eff } f(x, y, z)}=\left(1-D_{\lfloor(x, y, z)}\right) \sigma_{x}$

Given the biaxial and uniaxial tests performed in the calendering direction (CD) and the perpendicular direction (TD), this anisotropic damage evolution in which damage coupling and plane-shear damage have been neglected:

$$
\begin{aligned}
& \boldsymbol{D}_{\|(C D, T D, z)}=d_{c d \sigma d} \boldsymbol{C D} \otimes \boldsymbol{C D}+d_{t d t d} \boldsymbol{T D} \otimes \boldsymbol{T D}+d_{z z} z \otimes z \\
& \text { with } d_{t d t d}=d_{\infty}\left(1-e^{-\frac{\alpha}{\eta}}\right), d_{c d \sigma d}=d_{t d t d}+d_{0}, 0 \leq d_{0}, d_{\infty} \leq 1, \eta \geq 0, \alpha=\sqrt{\frac{I_{1}}{3}}
\end{aligned}
$$

where $d_{0}, d_{\infty}, \eta$ are three parameters to be fitted. At the difference of Miehe but in accordance with [9] this experimental damage depends on the strain $\alpha$. This matricial expression can be expressed in the material frame device:

$$
D_{\mid(x y, z)}=P^{t} D_{\mid(C D, T D, z)} P
$$

where $\boldsymbol{P}$ is the rotation matrix introduced in section 2.2.

In the TD direction (e.g. $\boldsymbol{x}$ corresponds to $\boldsymbol{T D}$ ) the damage is reduced to Miehe's expression (no damage in the initial state) and in the CD direction (e.g. $\boldsymbol{x}$ corresponds to $\boldsymbol{C D}$ ) the damage is affected by an initial value $d_{0}$ when it is not subjected to strain. This choice has been motivated by the material microstructural composition: due to the process, the macromolecular chains are preferably oriented in the calender direction and have been pre loaded in this direction, although in the transverse direction they are entangled. 
The damage function was determined using experimental results (from sequenced tensile tests in CD and TD directions) [9]. We consider results of Fig. 11, where we reduced experimental data to the sequences of loading curves of cycles 3, 6 and 9. Primary loading curves correspond to simple uniaxial tensile test and are denoted by $I_{1}, I_{2} I_{3}$ for the three primary loading. $\mathrm{I}_{1}^{z}, \mathrm{I}_{2}^{z}, \mathrm{I}_{3}^{z}$ are related to the three secondary loadings. From these data, we are able to determine discrete damage variables at stretches $\lambda_{\mathrm{x} 1}, \lambda_{\mathrm{x} 2}$ and $\lambda_{\mathrm{x} 3}$ by the expression:

$$
d_{x}\left(\lambda_{x \mathrm{xi}}\right)=\frac{\int_{0}^{\lambda_{\mathrm{xi}} r} \mathrm{I}_{\mathrm{i}}(\lambda) \mathrm{d} \lambda}{\int_{0}^{\lambda_{\mathrm{xi}}} \mathrm{I}_{\mathrm{i}}(\lambda) \mathrm{d} \lambda}, \mathrm{i}=1,2,3, \boldsymbol{X}=\boldsymbol{C D}, \boldsymbol{T D} .
$$

This energetic approach does not permit the reconstruction of the reloading curves. In fact these discrete coefficients only traduce a ratio of area under the curve and no the shape of the curve. For more complete analysis see reference [9].

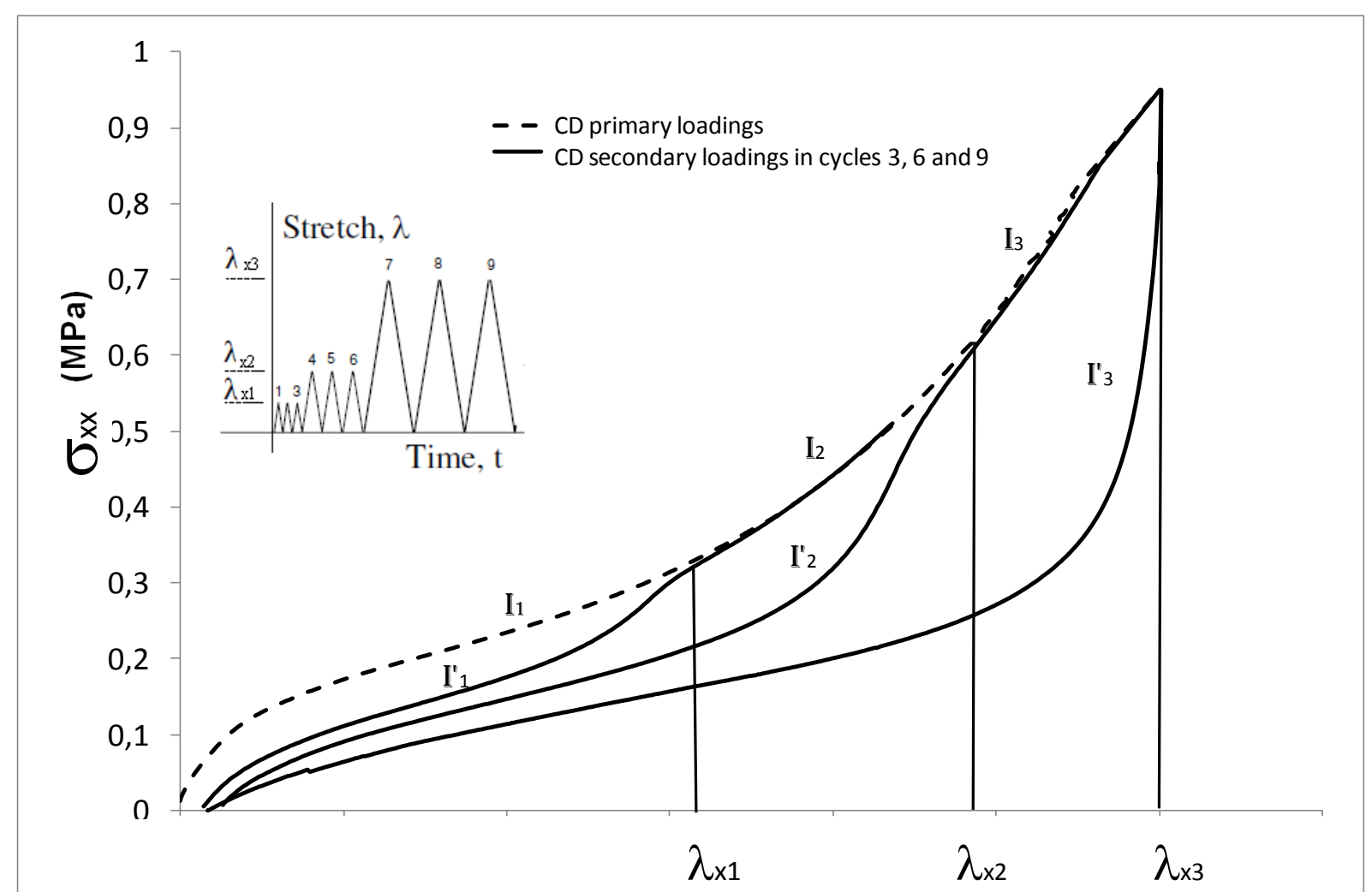

Fig. 11: Construction of damage evolution: evaluation of its discrete components

\subsection{Energy density parameter fit}

\subsubsection{Numerical estimation}

In this section, the SEBS stress evaluation is shown in terms of scripts written in Matlab® [34]. At each step of the test, DIC allows the definition of the full 2D in-plane Lagrangian strain tensor, a part of $\boldsymbol{F}$ is therefore known. Nevertheless we need one more information 
about the out-of-plane component for tests which are not uniaxial or test where calender direction is not similar with loading direction: $\lambda_{z}$.

For simple tensile tests experiments, and for any orientation of the reference material with respect to the reference loading, the only non-zero stress component (eq. 2) is $\sigma_{x x^{*}}$ This condition implies:

$$
\begin{aligned}
& \sigma_{x x}=\sigma_{x x}-\sigma_{y y}=\frac{1}{\lambda_{x} \lambda_{y} \lambda_{z}}\left(M_{1}\left(\lambda_{x}^{2}-\lambda_{y}^{2}\right)+M_{2}\left(\lambda_{x}^{-2}-\lambda_{y}^{-2}\right)\right) \\
& \quad \text { and } \\
& \sigma_{y y}-\sigma_{z z}=\frac{1}{\lambda_{x} \lambda_{y} \lambda_{z}}\left(M_{1}\left(\lambda_{y}^{2}-\lambda_{z}^{2}\right)+M_{2}\left(\lambda_{y}^{-2}-\lambda_{z}^{-2}\right)\right)=0 .
\end{aligned}
$$

In the case of biaxial tensile test, the only non-zero stress components are $\sigma_{x x}$ and $\sigma_{y y}$. These conditions imply:

$$
\begin{gathered}
\sigma_{x x}=\sigma_{x x}-\sigma_{z z}=\frac{1}{\lambda_{x} \lambda_{y} \lambda_{z}}\left(M_{1}\left(\lambda_{x}^{2}-\lambda_{z}^{2}\right)+M_{2}\left(\lambda_{x}^{-2}-\lambda_{z}^{-2}\right)\right) \\
\sigma_{y y}=\sigma_{y y}-\sigma_{z z}=\frac{1}{\lambda_{x} \lambda_{y} \lambda_{z}}\left(M_{1}\left(\lambda_{y}^{2}-\lambda_{z}^{2}\right)+M_{2}\left(\lambda_{y}^{-2}-\lambda_{z}^{-2}\right)\right) .
\end{gathered}
$$

For both experiments, the unknown out plane stretch $\lambda_{z}$ is therefore defined in term of stretches $\lambda_{x}, \lambda_{y}$ and material parameters $c_{10}, c_{01}, c_{11}$.

Analytical definition of strain/stress component for the specimen with two holes is not possible since the test is highly heterogeneous. The mechanical modeling is thus based on the Finite Element Method and integrates the mechanical behavior introduced in part 4.1.

\subsubsection{Numerical optimization}

Assuming parameters $\gamma=\left(c_{10}, c_{01}, c_{11}, d_{0}, d_{\infty}, \eta\right)$ known, theoretical stress / stretch curves were computed using procedure describe in section 4.2.1. These parameters can be adjusted to fit the experimentally-determined nominal stress versus stretch curves. The least squares technique is employed to best fit computed and experimental stress (see part (a) of Fig. 12). Let $m=\left(m_{1}, m_{2}, m_{3}, m_{4}\right)^{t}$ be the vector of values of a nominal stress measured at each step of the test. $m_{1}, m_{2}, m_{3}, m_{4}$ corresponds to $\sigma_{x x}$ for calender and transverse samples for uniaxial and biaxial loadings. The considered material model is represented by the strainenergy function from which the stress is calculated and written $\sigma_{x x}(\gamma)=\left(\sigma_{1 x x}(\gamma), \sigma_{2 x x}(\gamma), \sigma_{3 x x}(\gamma), \sigma_{4 x x}(\gamma)\right)$. The gap between experimental and numerical values is computed via a cost function written as the squared 2-norm 
$l(\gamma)=\frac{1}{2}\left(m-\sigma_{x x}(\gamma)\right)^{t}\left(m-\sigma_{x x}(\gamma)\right)$.

Hence, the minimization problem is given by

$\min _{\gamma \in U} J(\gamma)$

where $U$ is a set of constraints. The numerical optimization algorithm is Constrained Levenberg-Marquardt which is rather classical in the literature for solving identification problem. The parameters are such that $c_{10}, c_{01}, c_{11}, \alpha_{0}, \eta$ are positive, $0<d_{0}, d_{\infty}<1$, and $d_{x}\left(d, d_{\infty}, \eta, \alpha_{1}\right)=d_{\lambda_{w 1}}, d_{x}\left(d_{0}, d_{\infty}, \eta, \alpha_{2}\right)=d_{\lambda_{w 2}}, d_{x}\left(, d_{\infty}, \eta, \alpha_{3}\right)=d_{\lambda_{x y}}($ for $\boldsymbol{x}=\mathbf{C D}$ and TD see section 4.1).

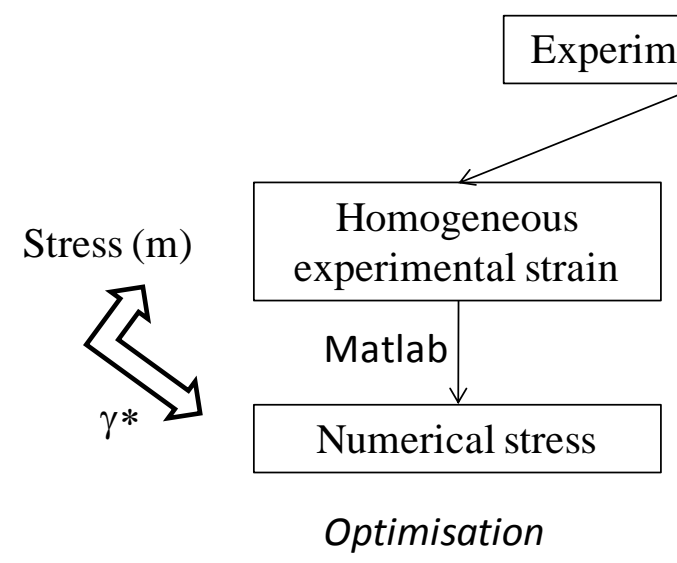

(a)

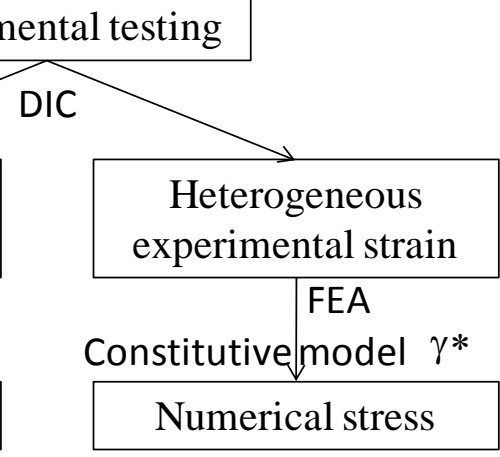

Validation

(b)

Fig. 12: Identification and validation

\section{Results and discussion}

\subsection{Homogeneous study}

After 10 iterations the minimization problem defined by (16) converges. The results of the identification process are listed in Table 3.

Table 3: Material parameters fitted from uniaxial and biaxial tests for sample in the calender and transverse directions

\begin{tabular}{|l|l|l|l|l|l|}
\hline$c_{10}(\mathrm{MPa})$ & $c_{01}(\mathrm{MPa})$ & $c_{11}(\mathrm{MPa})$ & $d_{0}$ & $d_{\infty}$ & $\eta$ \\
\hline 0.0002 & 0.0641 & 0.0004 & 0.255 & 0.4020 & 1.4 \\
\hline
\end{tabular}

To assess this identification procedure (accuracy and reliability), a sensitivity analysis has been made. The quality of this identification can be estimated via the ratio:

$$
S_{i}=\frac{\Delta \theta_{\mathrm{i}}}{\theta_{\mathrm{i}}} / \frac{\Delta J}{J}, i=1 . .6, \theta_{1}=\mathrm{c}_{10}, \theta_{2}=\mathrm{c}_{01}, \theta_{3}=\mathrm{c}_{11}, \theta_{4}=d_{0}, \theta_{5}=d_{\infty}, \theta_{6}=\eta \text {. }
$$


The lower is $S_{i}$ the more confidence we have in the identification process. Table 4 illustrates this issue. The parameter influencing the most $I$ (in eq. 16) is $c_{10}$ for the hyperelasticity and $\left(\eta, d_{0}\right)$ for the damage. Therefore both of these parameters mainly control the stress distribution.

Table 4: Sensitivity analysis

\begin{tabular}{|l|l|l|l|l|l|}
\hline$c_{10}(\mathrm{MPa})$ & $c_{01}(\mathrm{MPa})$ & $c_{11}(\mathrm{MPa})$ & $d_{0}$ & $d_{\infty}$ & $\eta$ \\
\hline 0.02 & 0.4 & 0.01 & 0.1 & 0.04 & 0.5 \\
\hline
\end{tabular}

The corresponding stress-stretch curves are shown in Figs. 13 and 14 for simple (ST) and biaxial tensile (BT) tests. Optimized parameters allowed the model to match the experimental data at least until 4.5 of axial stretch. This model is able to predict mechanical behavior: for the sample cut in the diagonal direction the fitted model gives satisfactory results (see Fig. 13). For the biaxial test, in the calender direction, the discrepancies appear beyond 4 of axial stretch. Moreover the model mark the difference in behavior depending on the loading direction: no transitory phase is observed for the material tested in the calender direction due to the pre stretched undergone by the material during the process (Fig. 14) The evolution of continuum damage is shown in Fig. 15 (see eq. 9). For the diagonal sample, initial damage is lower than the $\mathrm{CD}$ one but the asymptotic value is the same. It is interesting to note that during the process the material is stretched in the CD direction and is damaged to a corresponding value of $\mathrm{d}=0.2$. This damage corresponds to an equivalent dilatation $\lambda_{x}=2$ on the curve of the non pre-streched sample (see Fig. 15). On these curves, stars represent experimental discrete damage data at stretches $\lambda_{x 1}, \lambda_{x 2}, \lambda_{x 3}$.

Furthermore this modeling allows the numerical evaluation of the volume strain variation (see Fig. 16). From the knowledge of $\lambda_{z}$ numerically, the equation (1) conducts to the numerical volume strain variation for biaxial tensile tests (CD, TD) and uniaxial one (TD). We recall that, for the uniaxial tensile test performed in the calender direction (CD), the volume change is directly deduced from the measurement of in-plane stretch ratios associated with the assumption of transverse isotropy. It is clear that the biaxial test produces a greater volume strain variation than the uniaxial test (ratio of 10). In conclusion the model seems adequate to represent mechanical behavior of SEBS under homogeneous tests. 


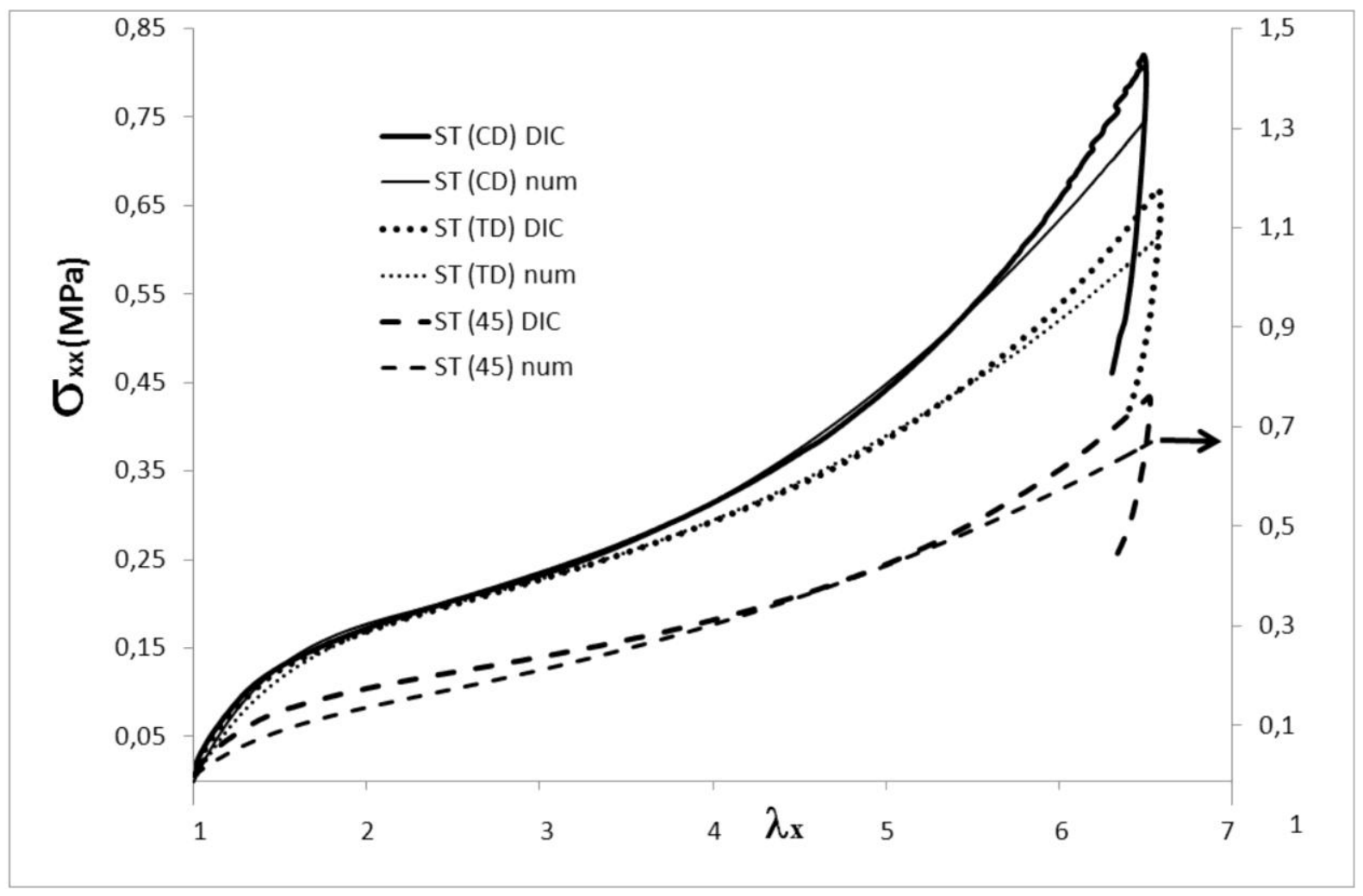

Fig. 13: Identification procedure based on uniaxial (ST) tests for CD and TD samples; Prediction for the sample cut in the diagonal direction $\left(45^{\circ}\right)$

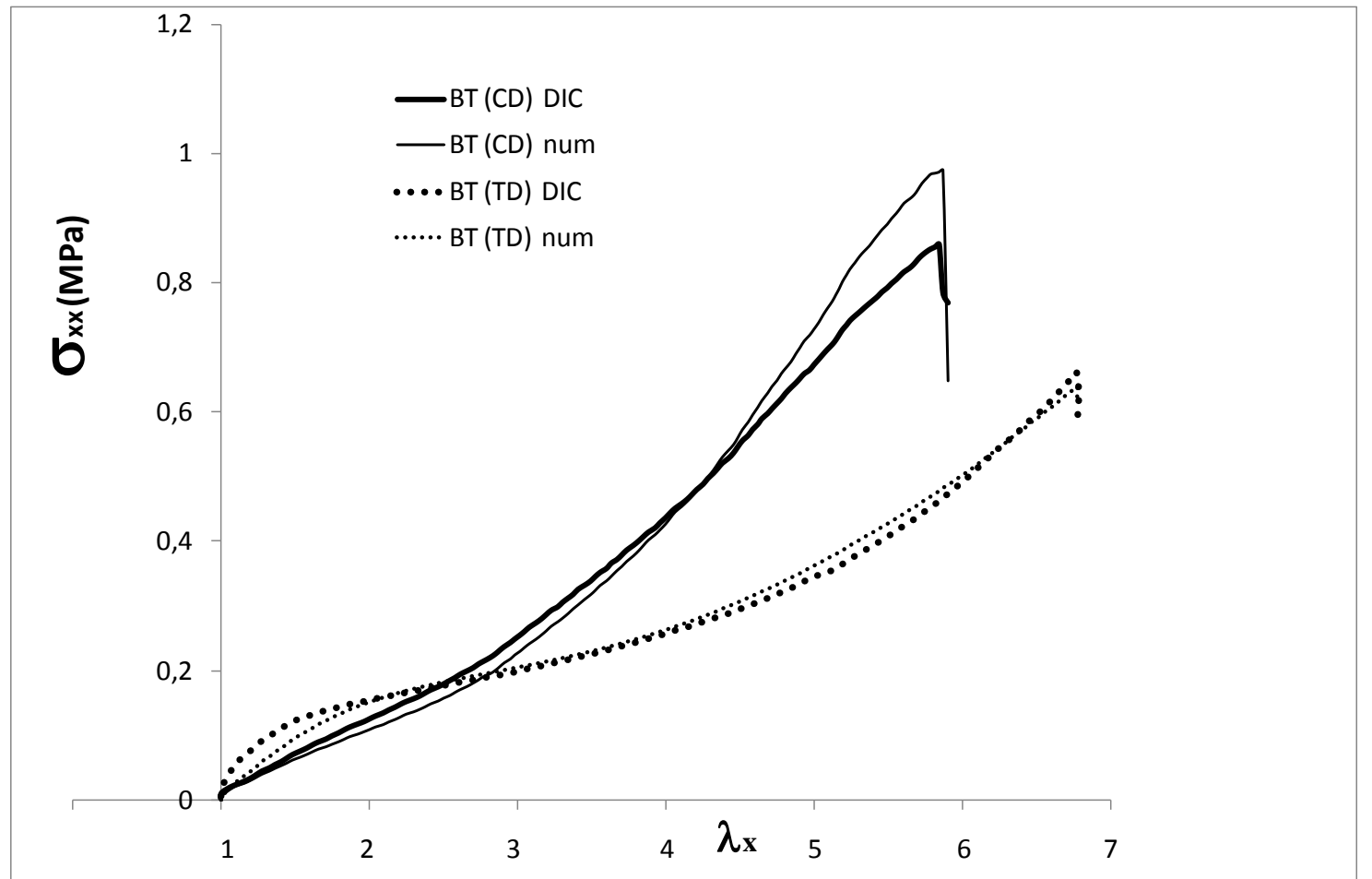

Fig. 14: Identification procedure based on biaxial (BT) tests for CD and TD samples 


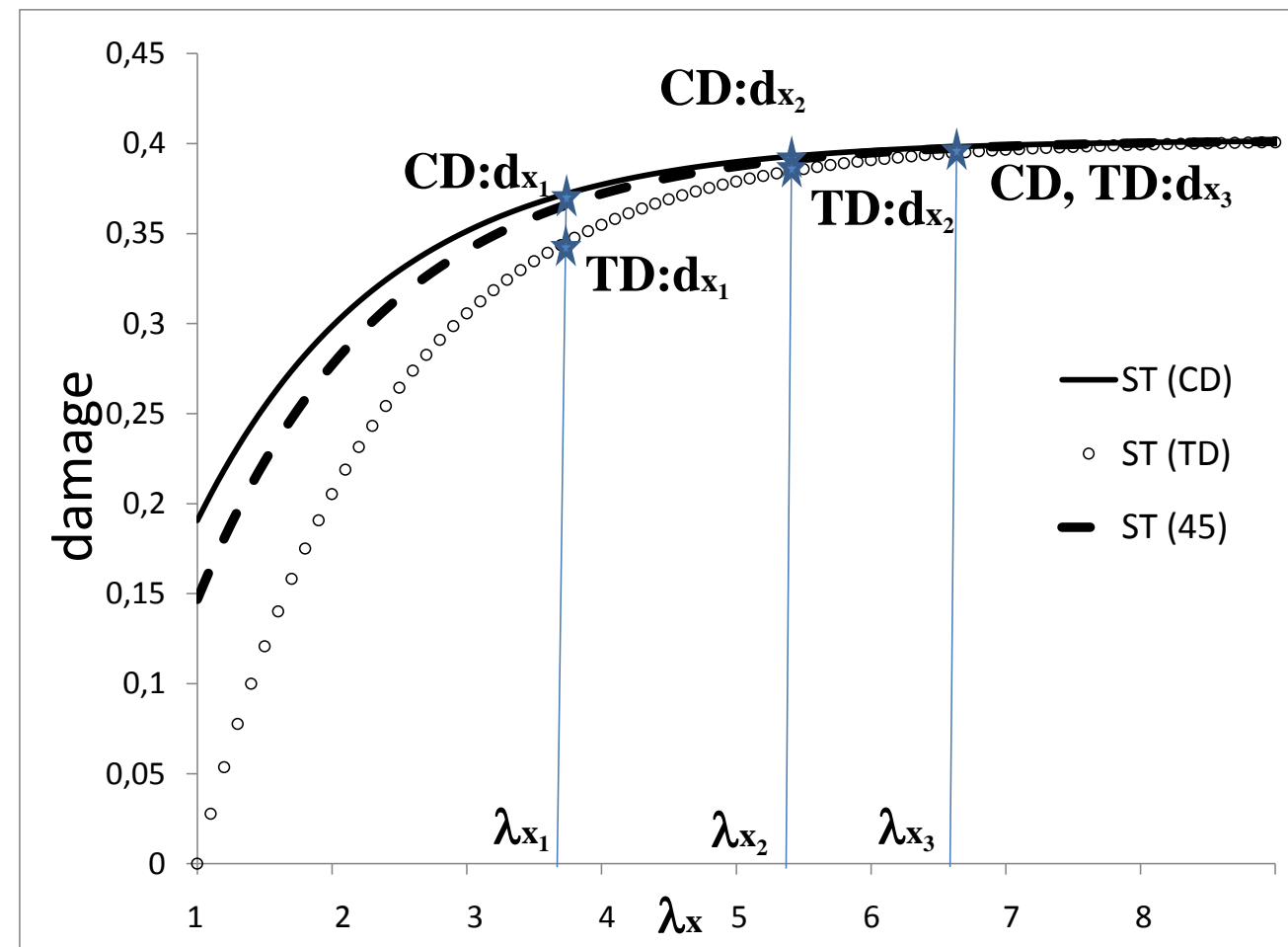

Fig. 15: Evolution of continuous damage for uniaxial test (ST) in the CD, TD and diagonal direction, discrete damage values at $\lambda_{x 1}, \lambda_{x 2}, \lambda_{x 3}$.

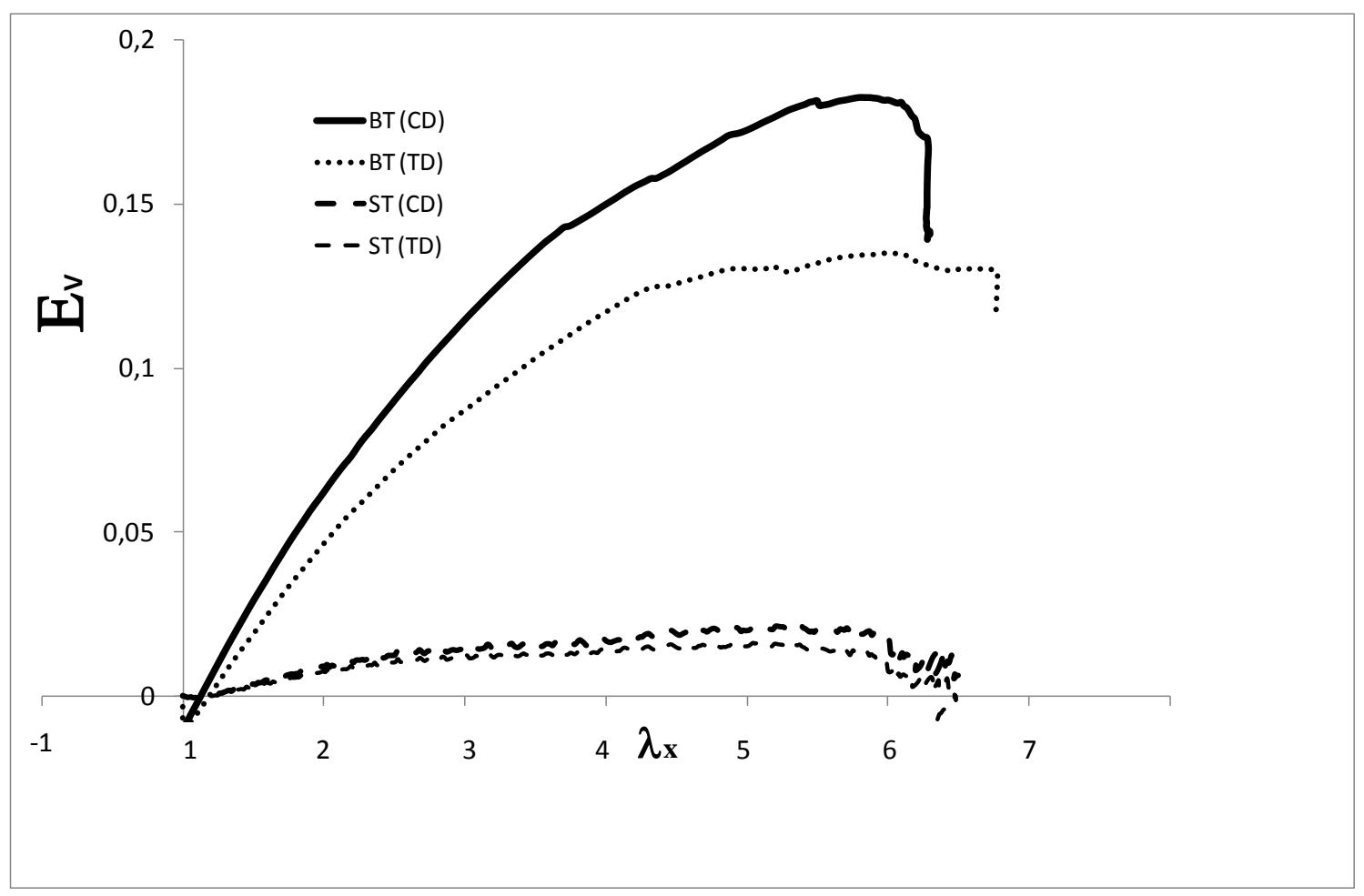

Fig. 16: Volumetric variation as a function of stretch ratio in an biaxial tensile test for CD and TD specimens. Comparison with uniaxial tensile tests. 


\subsection{Heterogeneous study}

To evaluate the capability of the model to describe a complete structure, a sample generating heterogeneous strain fields has been produced (see section 2.1). The numerical test was performed on ZeBuLON ([35]) with the constitutive model described in section 4.1 (already implemented in this software) and the fitted parameters given in Table 4 (see section 5.1). The finite element geometry (deformed and undeformed) and the boundary conditions can be found in Figs. 17-18. It has been modelled via 382 linear triangular elements. Displacement (corresponding to the load $F$ ) is applied on the upper and lower sides of the sample, under plane stress assumption. The test was conducted on the instrumented testing machine under the conditions described in section 2.2.2. For each node shown on Fig. 17, DIC gives access to the full in-plane strain components.
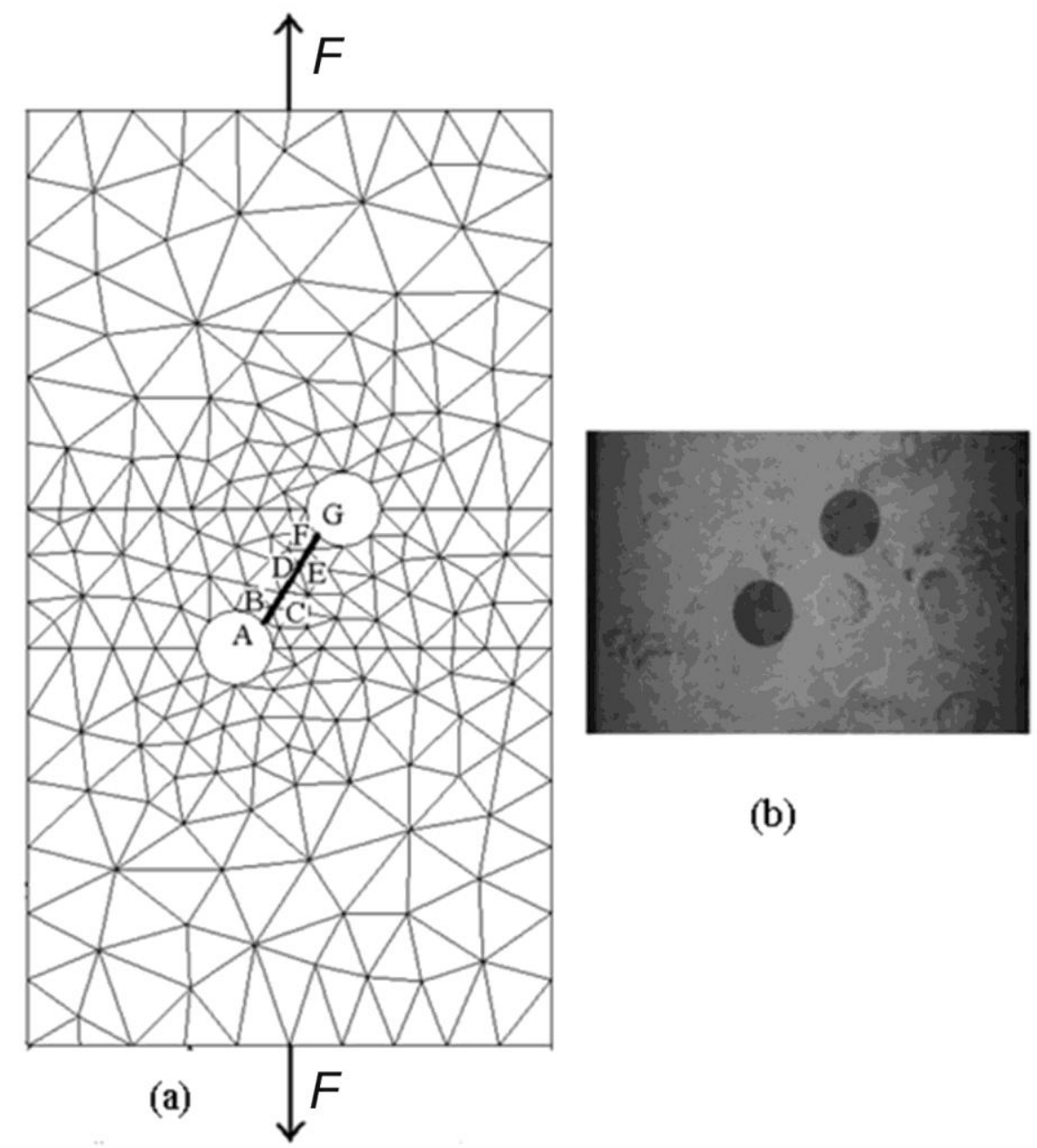

(b)

Fig. 17: FE Mesh and line from which the data are extracted (a); photograph for DIC (b) 
Fig. 18 (b) gives an image used for DIC at a force of $2.2 \mathrm{~N}$ and its corresponding (a) via the finite element simulation. It is clear that from a global point of view shape of the deformed sample corresponds.

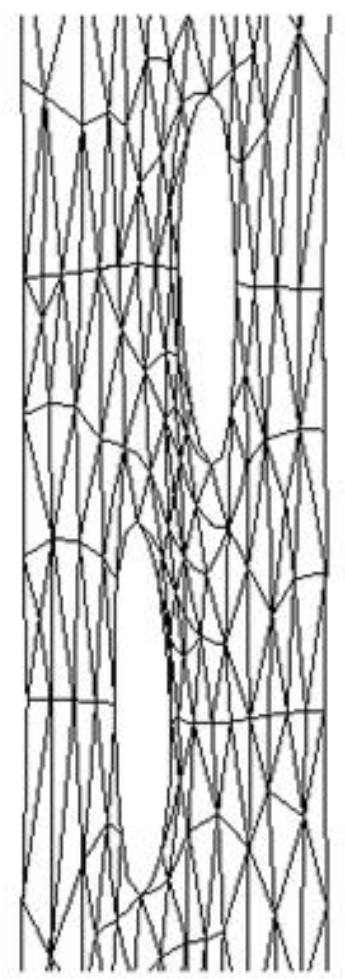

(a)

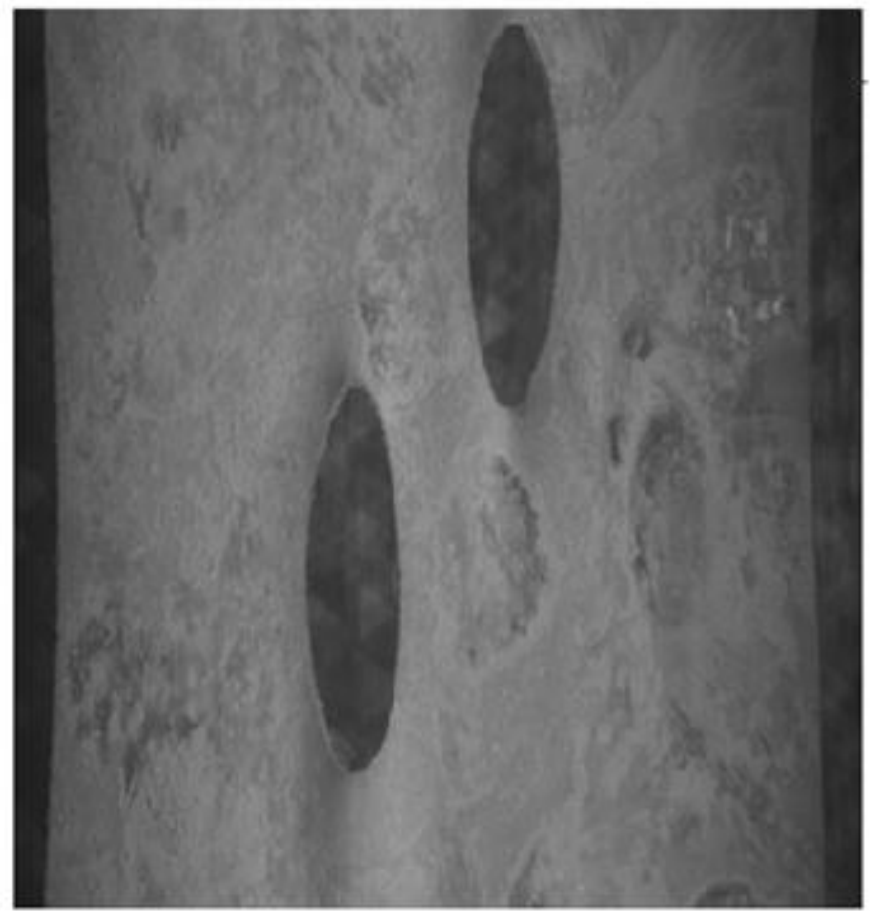

(b)

Fig. 18: Image of the deformed sheet for an applied force of $2.2 \mathrm{~N}$ (a) numeric (b) photograph

Experimental (DIC) and numerical (FEA) Lagrangian strains have been also compared along a line A-G (see Fig. 17) for different level of load. The chosen line extends from one hole to another and concentrated maximum shear points. The curves describing strains along the line is drawn in Fig. 19 for different load levels: strain deduced from DIC and FEA post processing corresponds. The numerical model with the fitted model parameters (see Table 4) gives a consistent response for the global behavior in comparison with the experimental one (Fig. 20). 


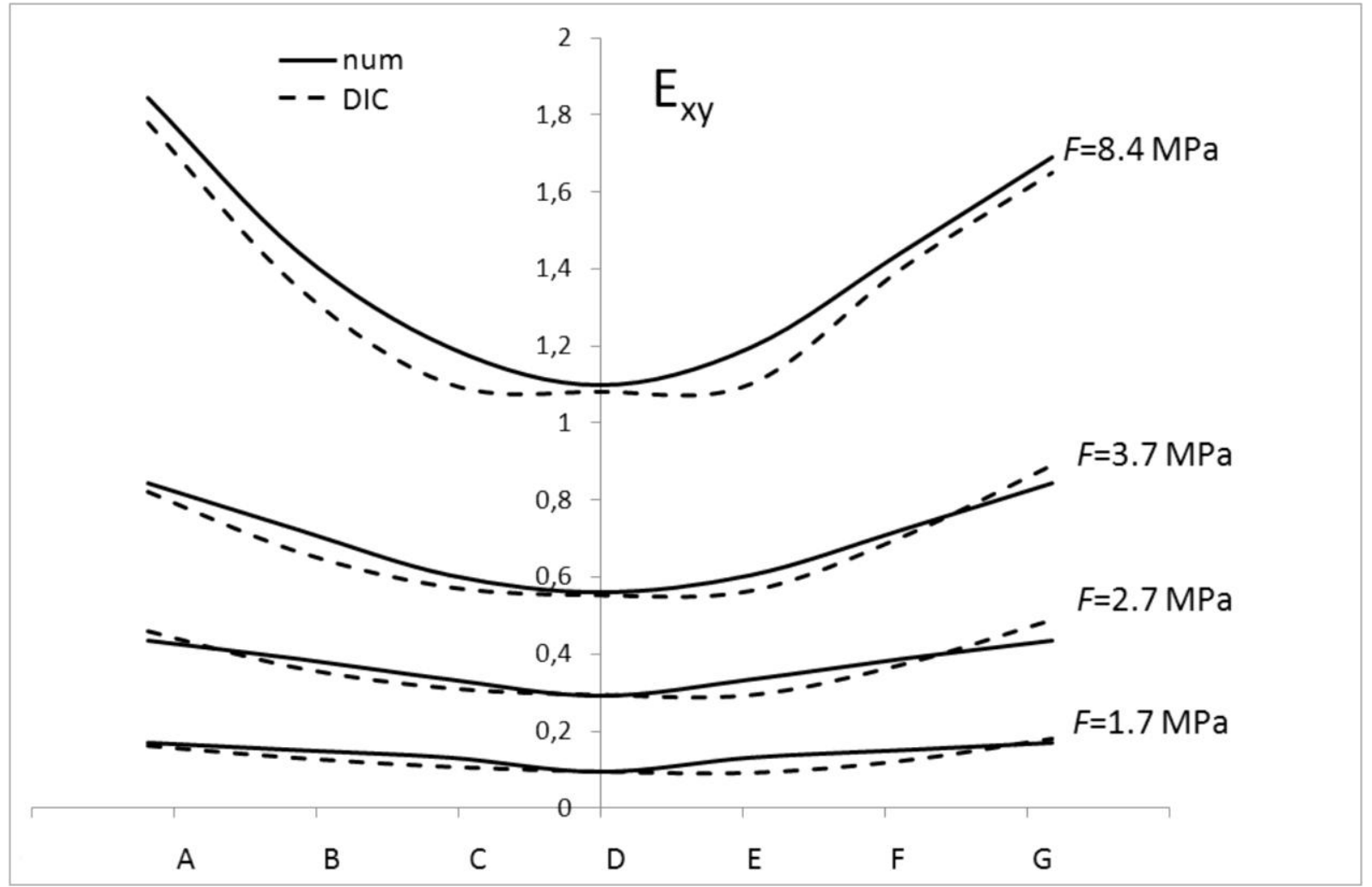

Fig. 19: Evolution of the shear Lagrangian strain component along the extended line between the two holes

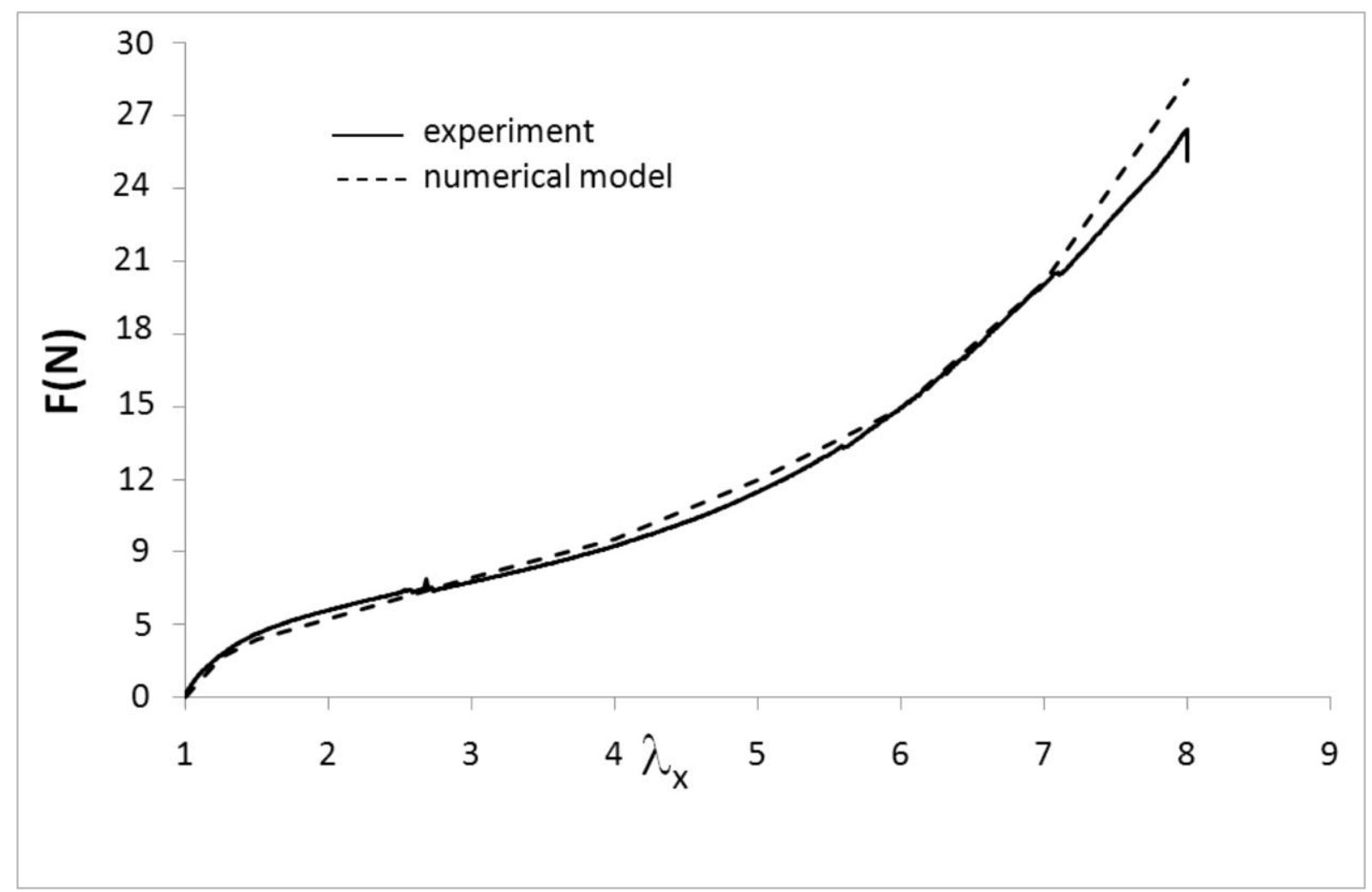

Fig. 20: Experimental and numerical stress-stretch response for the notched specimen 


\section{Conclusion}

In this paper mechanical investigations about SEBS behavior in link with the manufacturing process were conducted. A phenomenological model considering anisotropic damage function depending on the process (calender, transverse directions) associated with isotropic hyperelasticity and compressibility in large strain has been developed. DIC coupled with homogeneous standard tensile tests allows the identification of the 6 material parameters of the constitutive model for samples cut in the calender and transverse directions. This procedure allows the prediction of the local and global mechanical response of the material: validation on sample cut in the diagonal direction and on a rectangular specimen with two holes was successfully conducted.

The object of future works will concern the use of finite-element software to apply this inverse method to microstructural processes of deformation in a damageable area characterized by analytical and optical measurements (e.g. SEM, IR, or NMR).

\section{Acknowledgments}

We thank the AREVA and PIERCAN for providing the SEBS samples and for fruitful scientific discussions.

\section{References}

[1] Meier P, Lang M, Oberthür S. Plastic, Rubber and Composites 2005; 34(8):372-377

[2] Loew R, Meier P. Finite Elements in Analysis and Design 2006; 43:453-462

[3] Mullins L. Rubber Chemistry and Technology 1969; 42:339-362

[4] Meunier L, Chagnon G, Favier D, Orgéas L, Vacher P. Polymer Testing 2008; 27:765-777

[5] Mooney M. Journal of Applied Physics 1940; 11:582-592

[6] Bergström JS, Boyce MC. Mechanics of Materials 2000; 32:627-644

[7] Rivlin RS, Saunders DW. Philosophical Transactions of the Royal Society of London 1951; 243:251-288

[8] Miehe C. European Journal of Mechanics- A/Solids.1995; 14:697-720

[9] Chagnon G, Verron E, Gornet L, Marckmann G, Charrier P. Journal of the Mechanics and Physics of Solids 2004; 52:1627-1650

[10] Indukuri KK, Lesser AJ. Polymer 2005; 46:7218-7229 
[11] Aggarwal SL. Polymer 1976; 17: 938-956

[12] Honeker CC, Thomas EL. Chemistry of Materials 1996; 8:1702-1714

[13] Xu ZW, Liang YC, Dong S, Cao YZ, Zhao TQ, Wang JH, Zhao QL. Ultramicroscopy 2005; 105:72-78.

[14] Christman A, Ienny P, Quantin JC, Caro-Bretelle AS, Lopez-Cuesta JM. Polymer 2011; 52(18):4033-4044

[15] Giton M, Bretelle AS, Ienny P. Strain 2006; 42:291-297

[16] Mullins LJ. Journal of Rubber Research 1947; 16:275-289

[17] Le Cam JB. Rubber Chemistry and Technology 2010; 83:247-269

[18] Le Cam JB, Toussaint E. Mechanics of Materials 2009; 41:898-901

[19] Rubinstein M, Panyukov S. Macromolecules 2002; 35:6670-6686.

[20] Gent AN, Thomas AG. Journal of Polymer and Science 1958; 28:625-637

[21] Hart-Smith LJ. Journal of Applied Mathematics and Physics 1966; 17:608-626

[22] Lambert-Diani J, Rey C. European Journal of Mechanics and Solids 1999; 18:10271043

[23] Ogden RW, Saccomandi G, Segura I. Computational Mechanics 2004; 34:484-502

[24] Miehe C. International Journal for Numerical Methods in Engineering 1994; 37:19812004

[25] Diani J, Brieu M, Vacherand JM, Rezgui A. Mechanics of Material 2004; 36:313-321

[26] Spencer AJM, Zheng QS. Journal of the Mechanical and Physics of Solids 2001; 49: 2455-2458

[27] Mullins LJ. Rubber Chemistry and Technology 1969; 42:339-362

[28] Harwood JAC, Mullins L, Payne AR. Journal of Applied Polymer1965; 9:3011-3021

[29] Diani J, Fayolle B, Gilormini P. European Polymer Journal 2009; 45(3):601-612

[30] Mullins L, Tobin NR. Rubber Chemistry and Technology 1957; 30:555-571

[31] Johnson MA, Beatty MF. Continuum Mechanics and Thermodynamics 1993; 5:301-

[32] Simo JC. Computer Methods in Applied Mechanics and Engineering 1987; 60:153173

[33] Lemaitre J, Chaboche JL. Journal of Applied Mechanics 1978 ; 2:167-189

[34] MATLAB 2003; Version 6.5, The Math Works Inc

[35] Besson J, Leriche R, Foerch R, Cailletaud G. Revue européenne des éléments Finis $1998 ; 7: 567-588$ 


\section{LIST OF FIGURES}

Fig. 1. Anisotropy induced by the process

Fig. 2. Useful dimensions of specimens: for uniaxial (a), biaxial (b) compression loadings (c) and heterogeneous test (d) (quotations in $\mathrm{mm}$, thickness $0.6 \mathrm{~mm}$ )

Fig. 3. Material and device frames

Fig. 4. Uniaxial stress as function of measured stretch ratios (measured by DIC and calliper device)

Fig. 5. Uniaxial stress versus stretches ratios for SEBS manufactured by a calender process $(\mathrm{CD}, \mathrm{TD})$ and by dip coating process

Fig. 6. Definition of the two-dimensional digital extensometer (CS: correlation size; GS: grid step

Fig. 7. Stress-Stretch response during three cycles of loading-unloading at 3.7, 5.3 and 6.6 of stretch (for CD and TD samples)

Fig. 8. Volumetric variation as a function of mechanical cycling for a CD sample

Fig. 9. Axial stress versus stretches for CD and TD specimens for a biaxial loading

Fig. 10. Load-displacement curve in oedometric compression

Fig. 11. Construction of damage evolution: evaluation of its discrete components

Fig. 12. Identification and validation

Fig. 13. Identification procedure based on uniaxial (ST) tests for CD and TD samples; Prediction for the sample cut in the diagonal direction $\left(45^{\circ}\right)$

Fig. 14. Identification procedure based on biaxial (BT) tests for CD and TD samples

Fig. 15. Evolution of continuous damage for uniaxial test (ST) in the CD, TD and diagonal direction, discrete damage values at $\lambda_{x 1}, \lambda_{x 2}, \lambda_{x 3}$

Fig. 16. Volumetric variation as a function of stretch ratio in an biaxial tensile test for $\mathrm{CD}$ and TD specimens

Fig. 17. FE Mesh and line from which the data are extracted (a); photograph for DIC (b)

Fig. 18. Image of the deformed sheet for an applied force of $2.2 \mathrm{~N}$ (a) numeric (b) photograph

Fig. 19. Evolution of the shear Lagrangian strain component along the line joining the 
two holes

Fig. 20. Experimental and numerical stress-stretch response for the notched specimen 


\section{LIST OF TABLES}

Table 1. Data parameter used by the DIC Process

Table 2. Variation of the bulk modulus with the hydrostatic pressure

Table 3. Material parameters fitted from uniaxial and biaxial test for sample in the calender and transverse directions

Table 4. Sensitivity analysis 